د. فاتن هادي الحربي

المجلد السابع والعشرون العلدد (2)، مارس 2021م

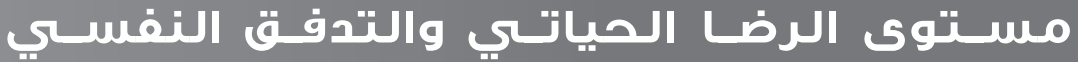

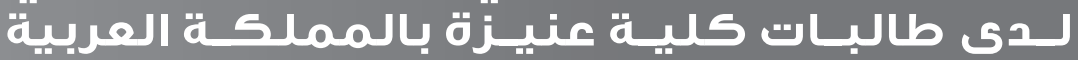 السمودية
}

الاسـتلام : 25 / مارس / 2021

التحكيــــ

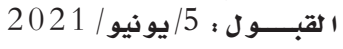

د. فاتن هادي الحربي)(1*)

(c) 2021 University of Science and Technology, Yemen. This article can be distributed under the terms of the Creative Commons Attribution License, which permits unrestricted use, distribution, and reproduction in any medium, provided the original author and source are credited.

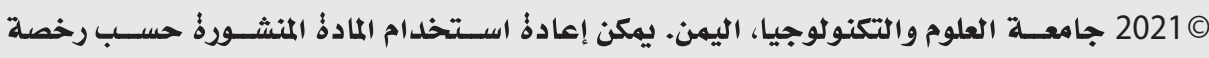
مؤسسة المشاع الإبداعي شريطة الاستشهاد بالمؤلف والمجلة. 
مستوى الرضا الحياتي والتدفق النفسي لدى طالبات كلية عنيزة بالمملكة العربية السعودية النفية

الملخص:

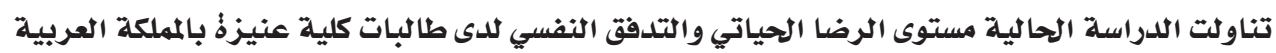

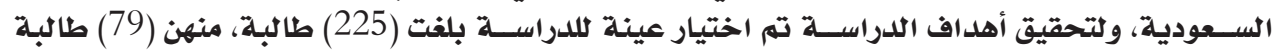

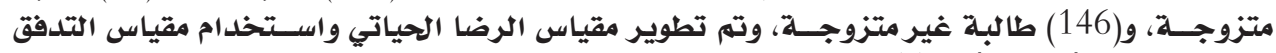

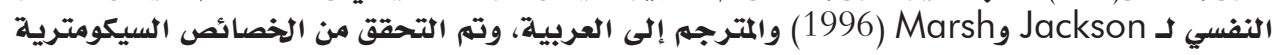

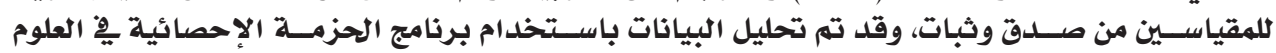

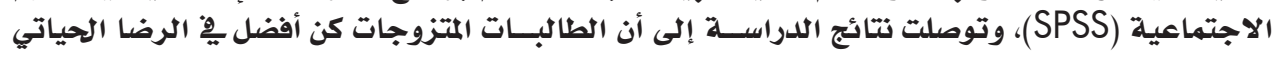

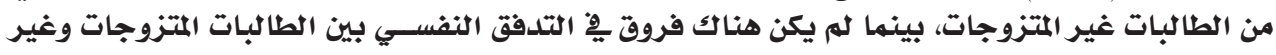

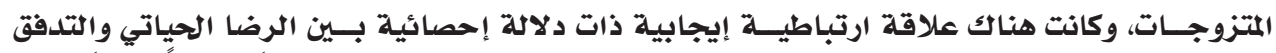

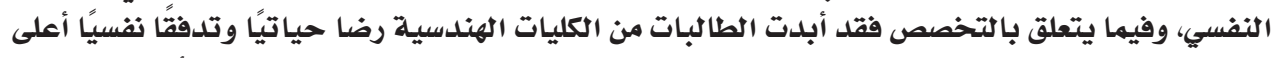

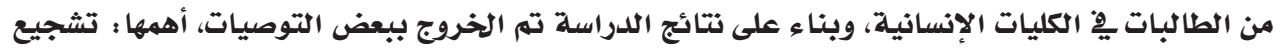

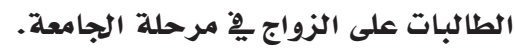

الكلمات المفتاحية : التدفق النفسي، الرضا الحياتي، الزواج والتخصص. 


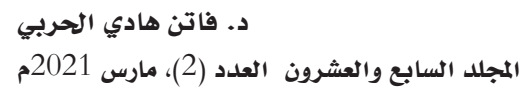

\title{
Level of Life Satisfaction and Psychological Flow among Female Students at Unaizah College, Saudi Arabia
}

\begin{abstract}
:
This study investigated the level of life satisfaction and psychological flow among female students at Unaizah College in Saudi Arabia. To achieve this, a measure for life satisfaction was developed and the Arabic version of the psychological flow measure, developed by Marsh and Jackson (1996), was adopted. After verifying the psychometric properties of the two scales, they were administered to a sample of 225 female students $(79$ married and 146 unmarried). The collected data was processed and analyzed by using the SPSS. The findings revealed that married students had more life satisfaction than unmarried ones, but there were no differences between them regarding the psychological flow. There was also a statistically significant positive correlation between life satisfaction and the psychological flow. Regarding specialization, students of engineering colleges showed more life satisfaction and psychological flow than students of human colleges. The study concluded with a set of recommendations, including encouraging female students to get married while at college.
\end{abstract}

Keywords: psychological flow, life satisfaction, marriage and specialization. 


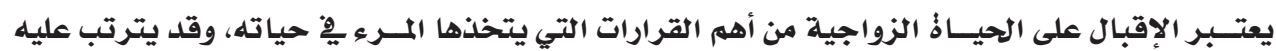

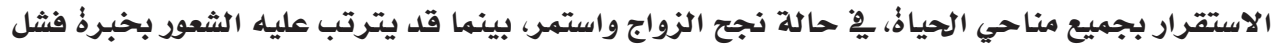

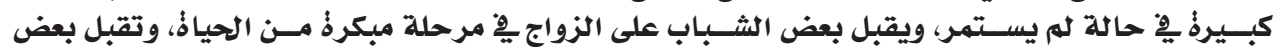

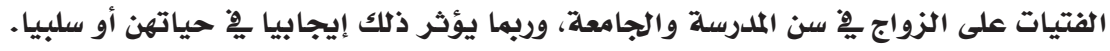

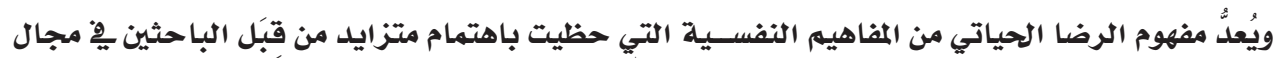

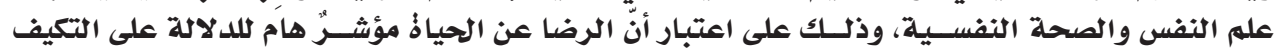

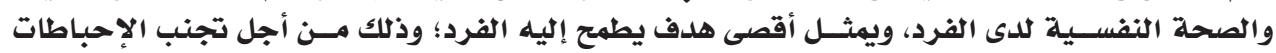

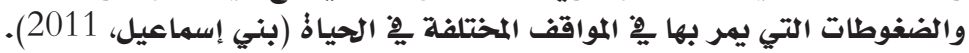

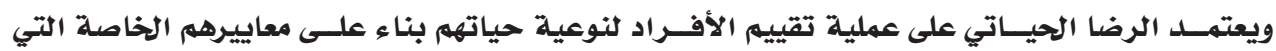

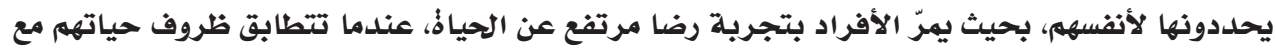

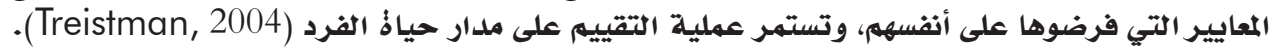

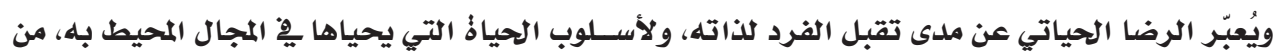

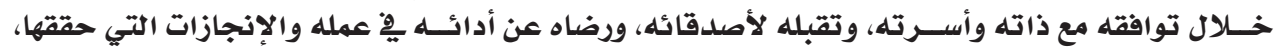

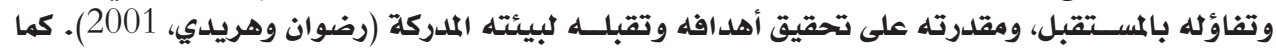

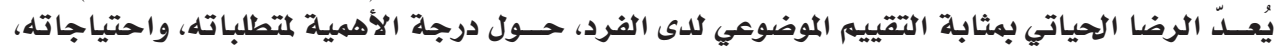

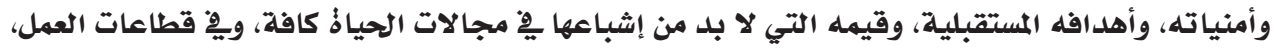

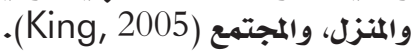

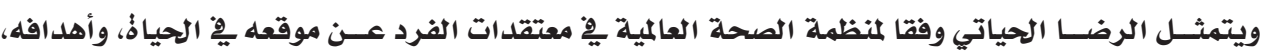

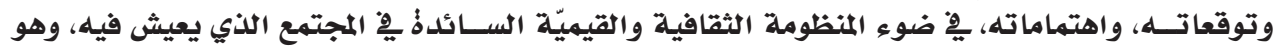

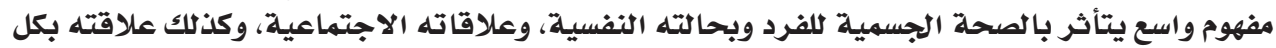
مكونات البيئة التي يعيث فيها ئرائ (عيسى ورشوان، 2006).

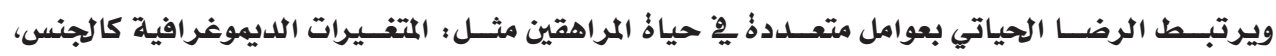

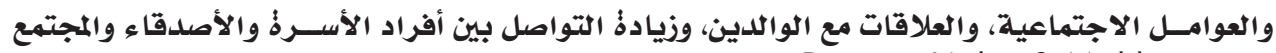
.(Proctor, Linley \& Maltby, 2008) ويشـير Huebner Suldo (2006) إلى أنّ الرضــا الحيـاتي للدى المراهقين يرتبط بطريقة التنشـئة

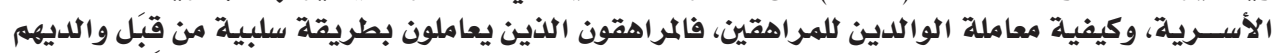

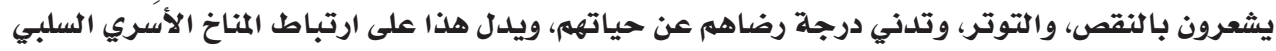
بتدني درجة الرضا الحياتي لدى والدون المراهقين.

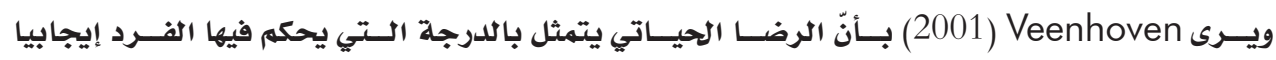

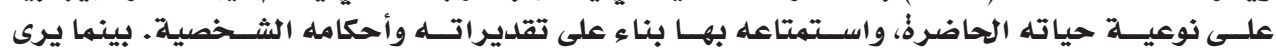

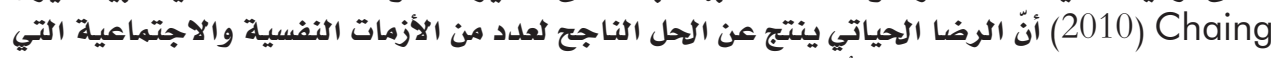

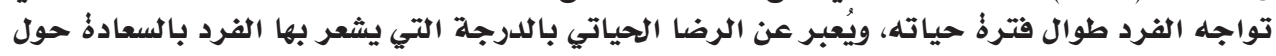
وضعه العام مِِِ الحياةً.

ويقيس الرضا الحياتي بناء على نظرية المقارنة الاجتماعية الأبعاد الآتية :

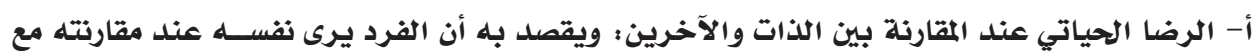
الآخرين أفضل حالا منهم.

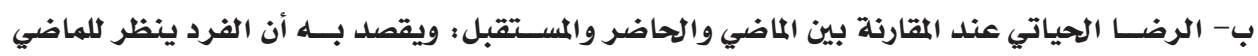

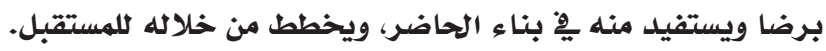




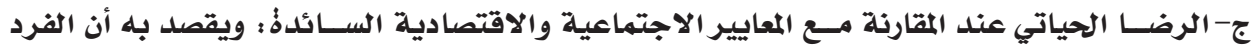

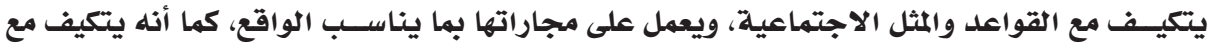
الظروف الاقتصادية والمالية التي يعيث بها التها (Fastingers \& Easterlin, 2001).

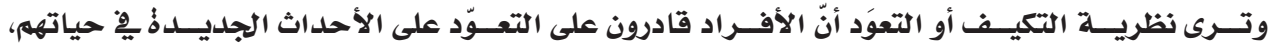

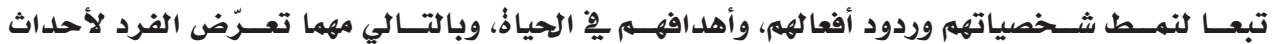

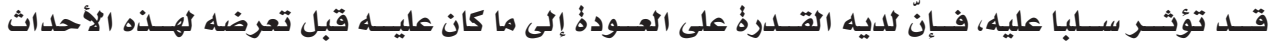

.(Diener \& Rahtz, 2009)

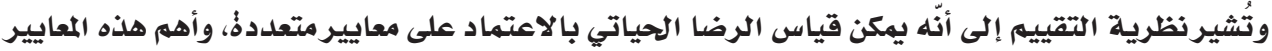

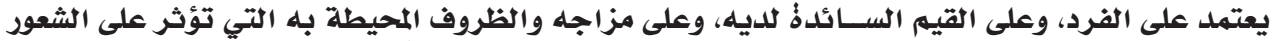

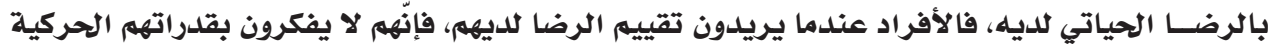

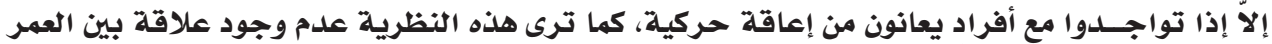

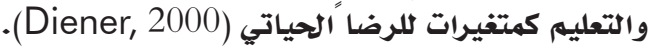

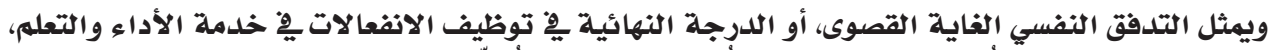

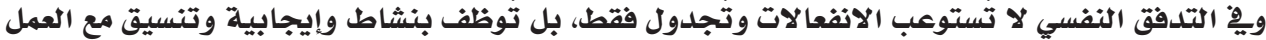

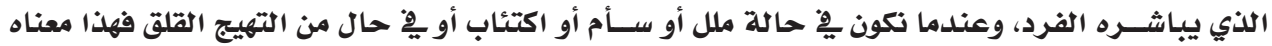

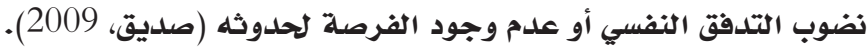

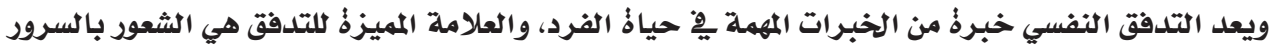

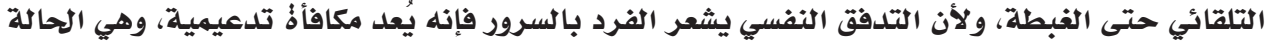

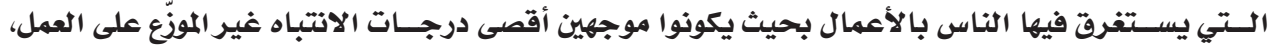

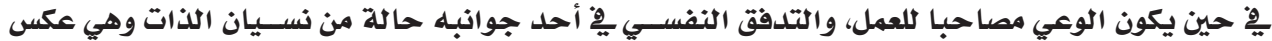

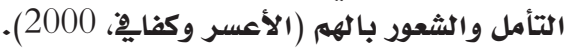

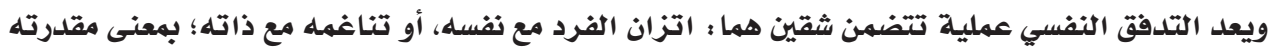

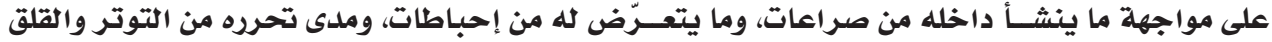

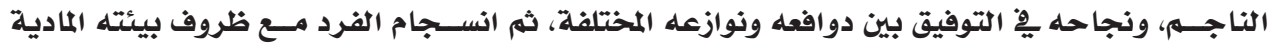

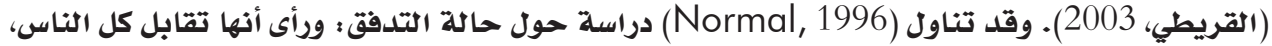

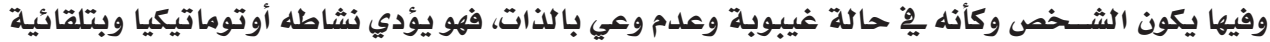

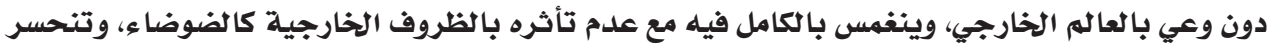

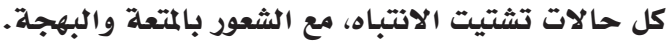

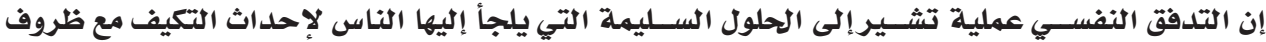

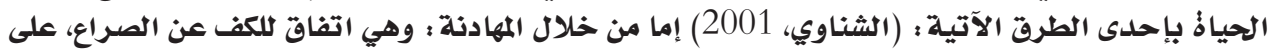

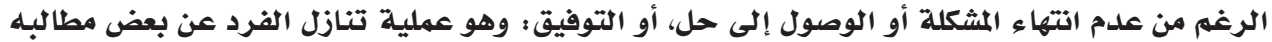

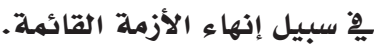

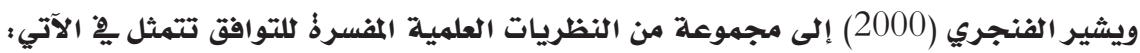
1. نظرية التحليل النفسـي : التدفق النفسـي هو قدرةٌ الفرد على أن يقوم بعملياته العقلية والنفسية

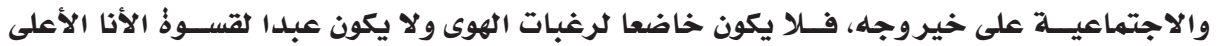

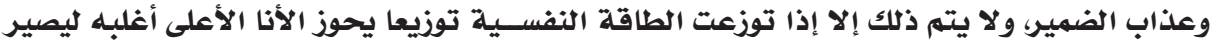

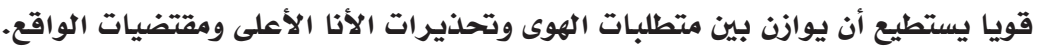

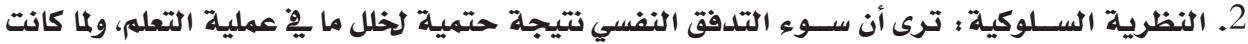

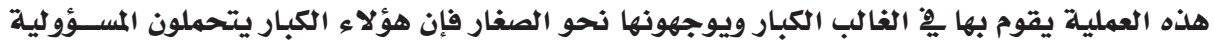

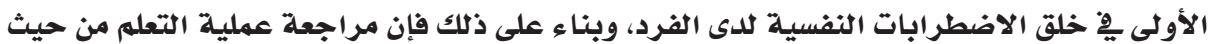

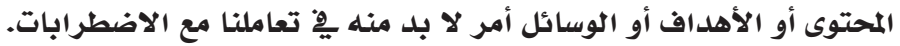




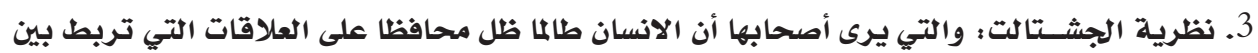

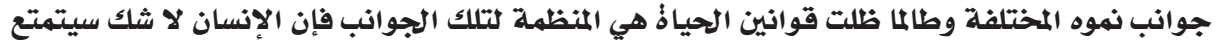

بالصحة النفسية.

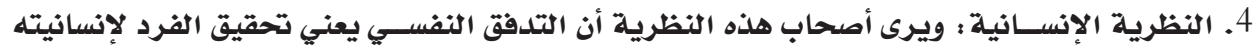

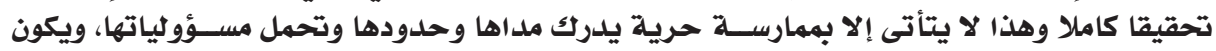

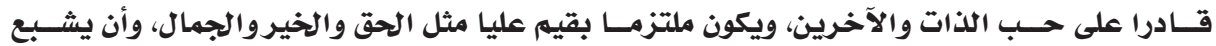
حاجاته الفسيولوجية والنفسية إشباعا متزنا. الدراسات السابقة:

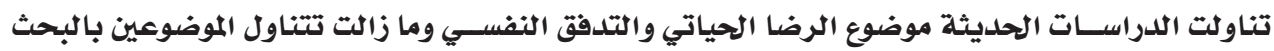

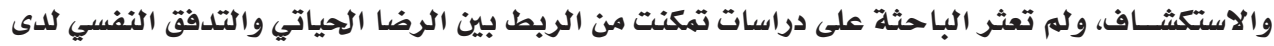

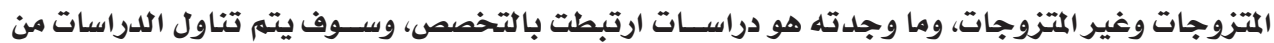

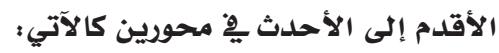

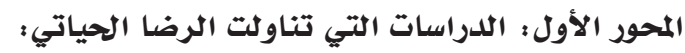
قام سليمان (2003) بلدراسة حول الرضا الحياتي وعلاقتته بتقدير الذات للدى ملديري المدارس الدات الحكومية

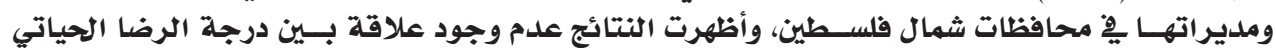

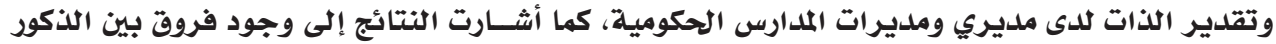

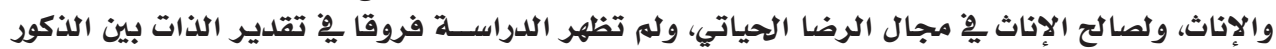
والإناث.

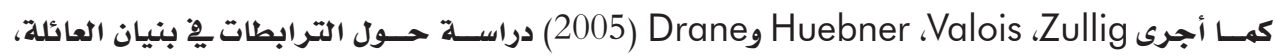

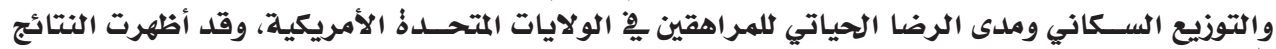

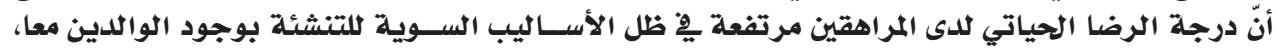

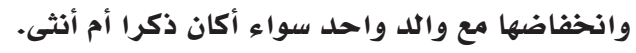

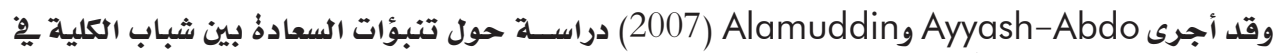

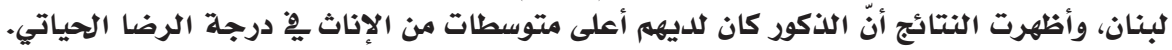

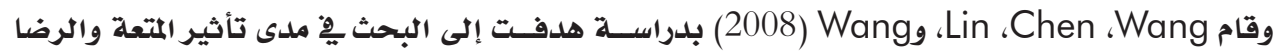

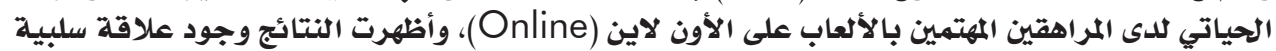

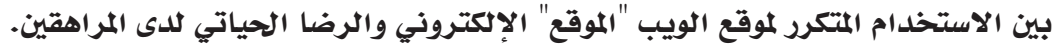

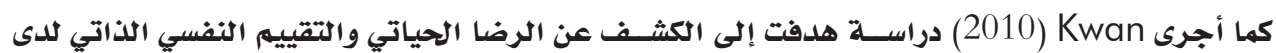

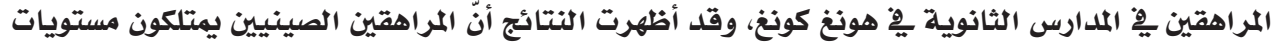

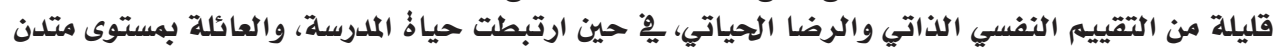

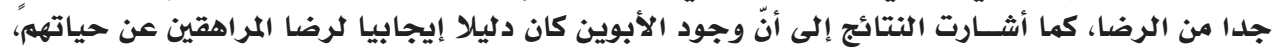

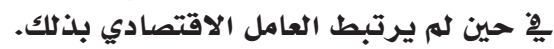

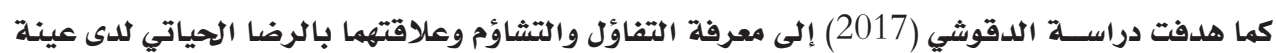

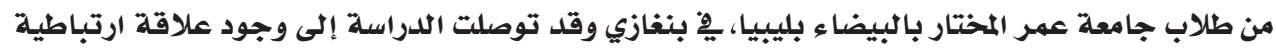

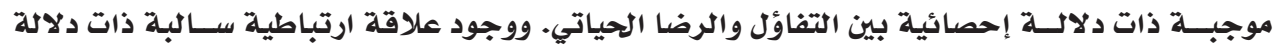

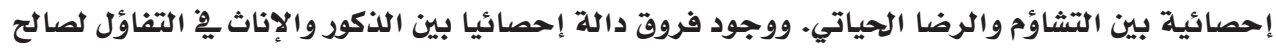

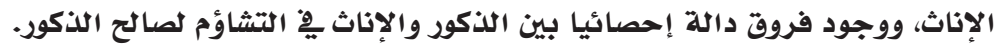




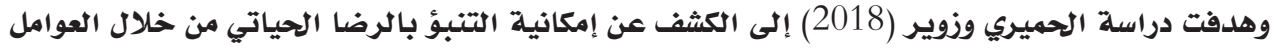

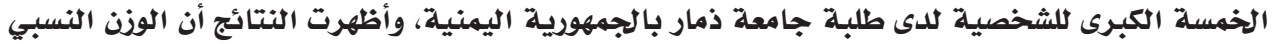

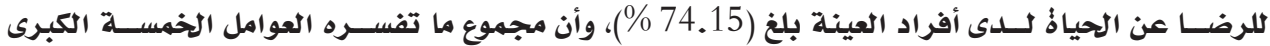

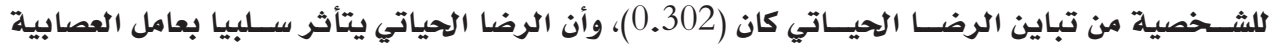

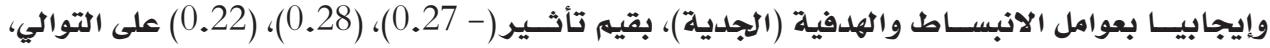

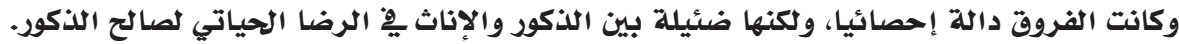

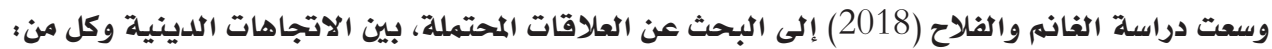

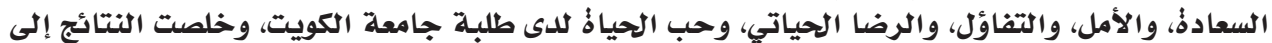

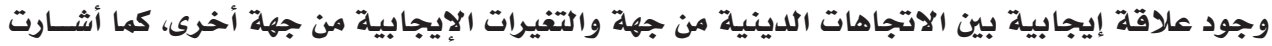

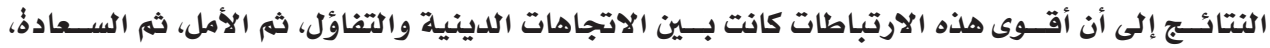

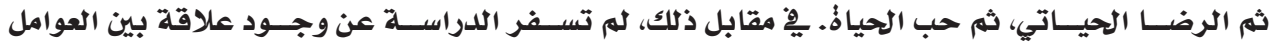

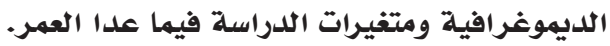

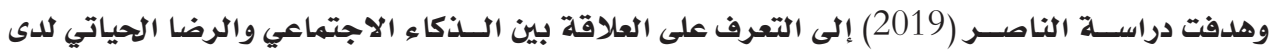

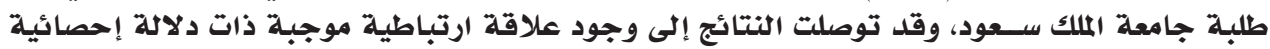

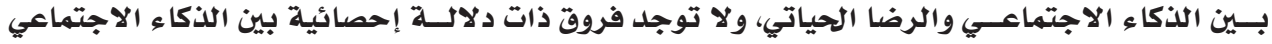

والرضا الحياتي بحسب النوع (ذكر، أنثى)، أو بحسب التهائ التخصص (تخصاء (تصصات علمية، تخصصات إنسانية).

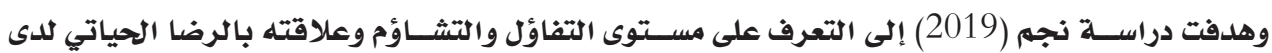

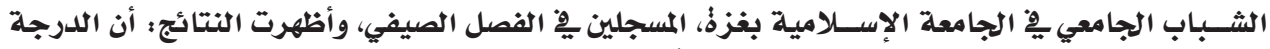

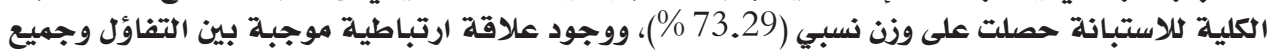

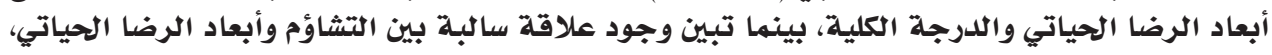

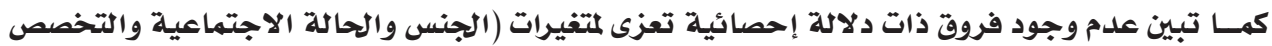
والمستوى الدراسي والمعلد إنود التراكمي).

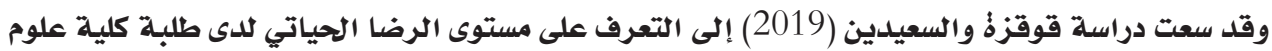

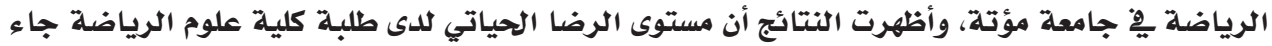

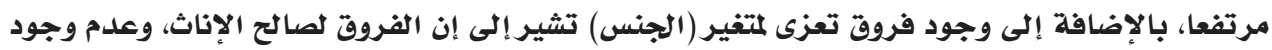

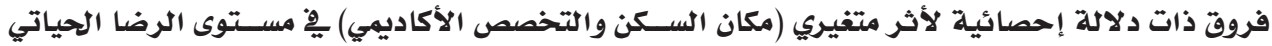

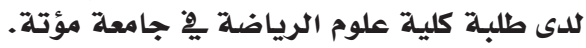

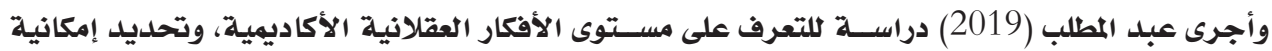

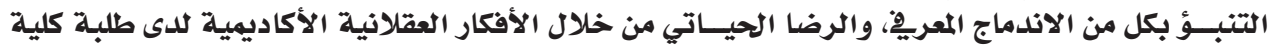

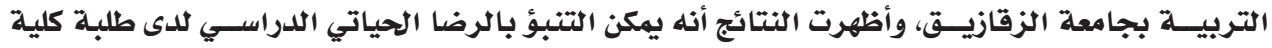

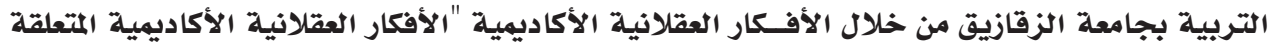

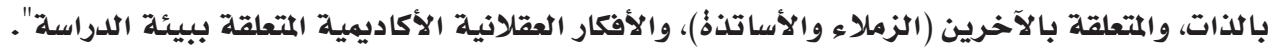

$$
\text { المحور الثاني: الدراسات التي تناولت التدفق النفسي }
$$

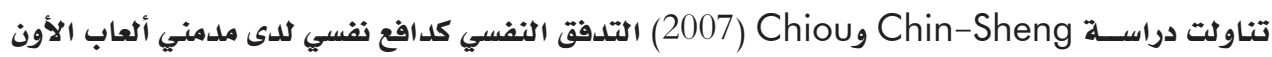

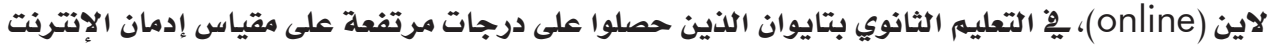

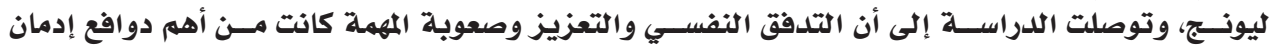

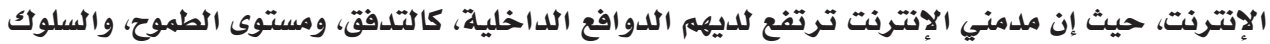

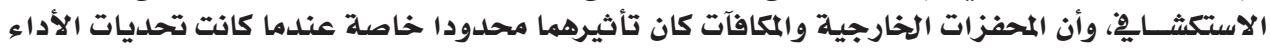




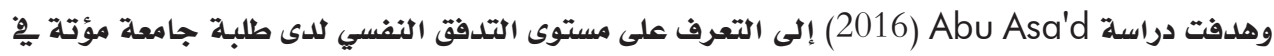

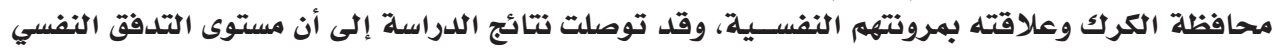

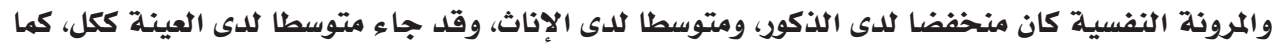

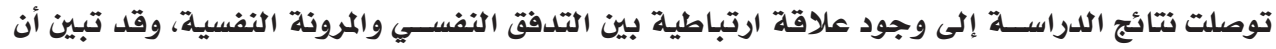

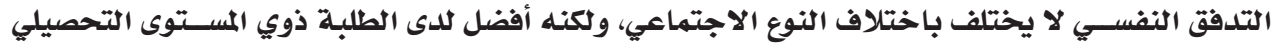

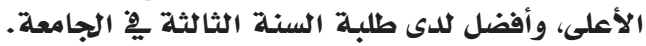

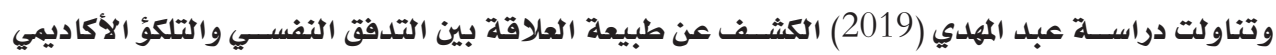

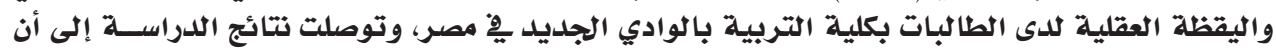

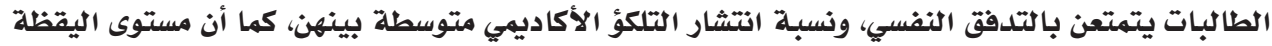

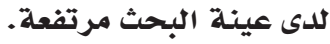

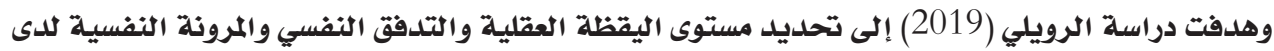

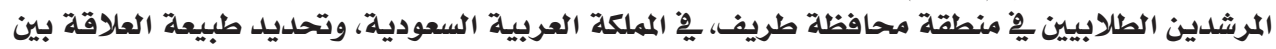

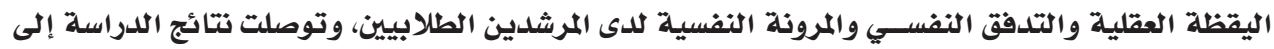

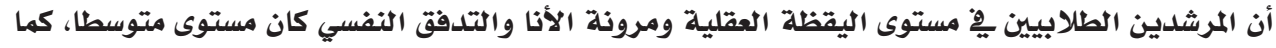

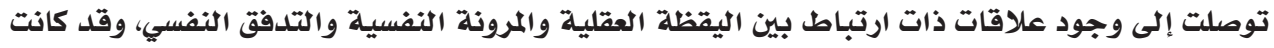

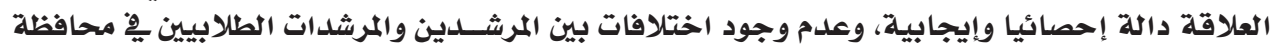

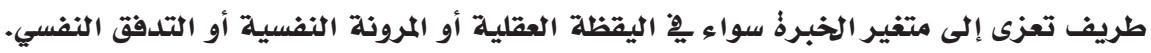

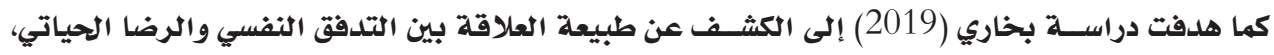

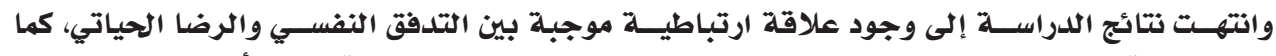

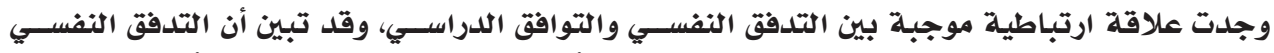

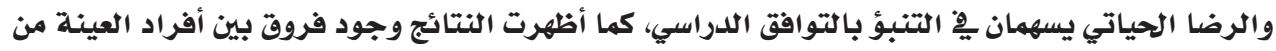

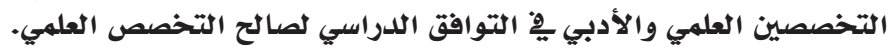

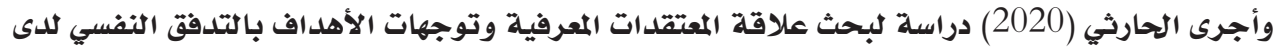

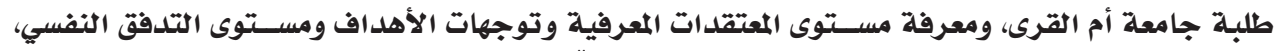

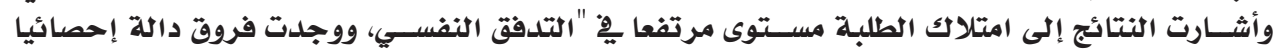

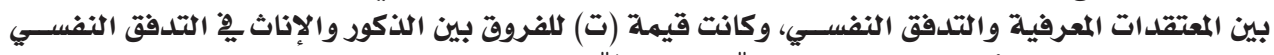

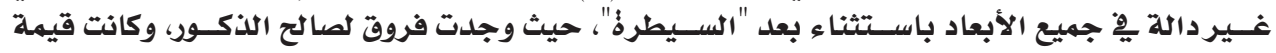

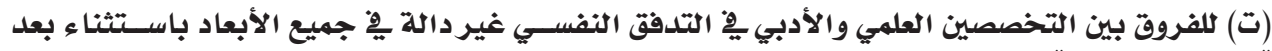

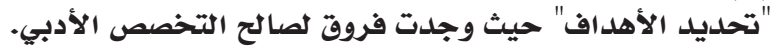

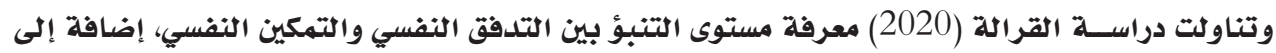

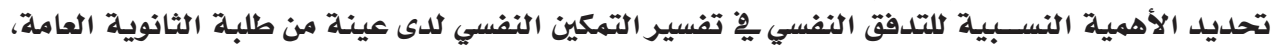

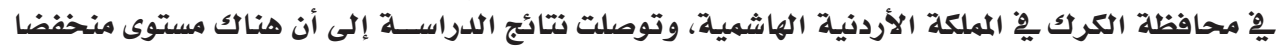

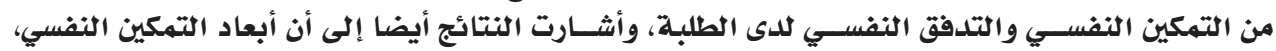

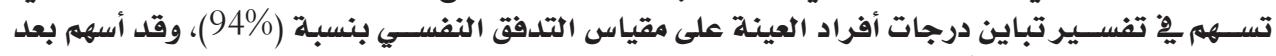

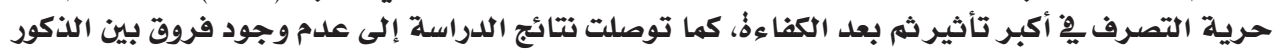

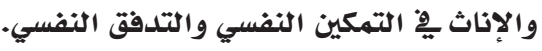

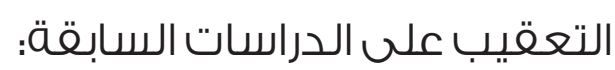

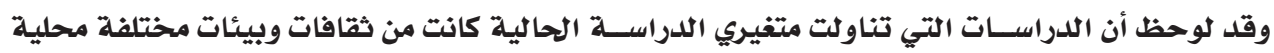

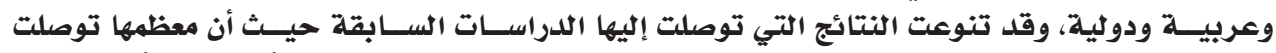

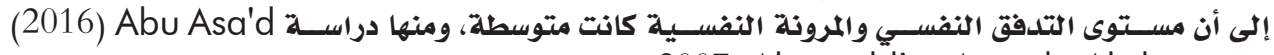

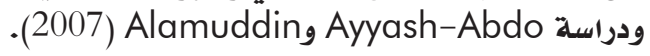




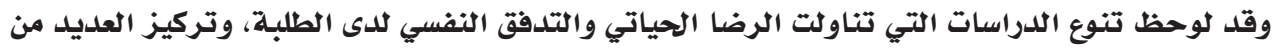

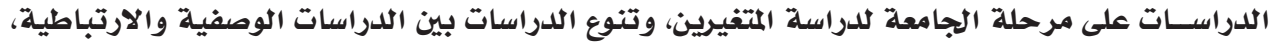

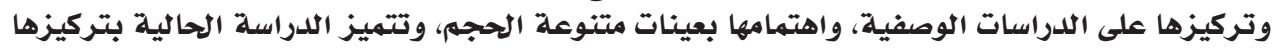

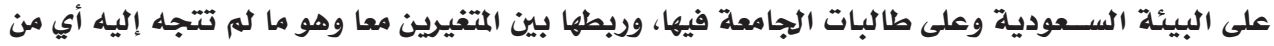

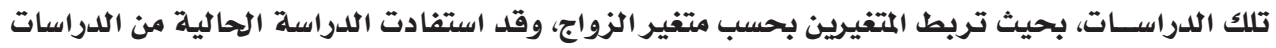

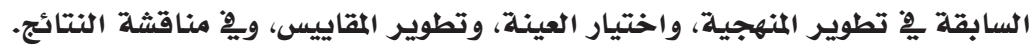

$$
\text { مشكلة الدراسة: }
$$

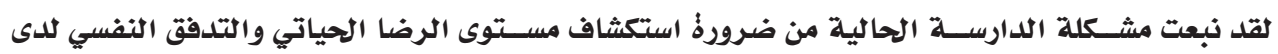

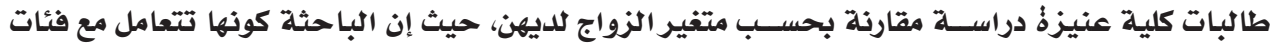

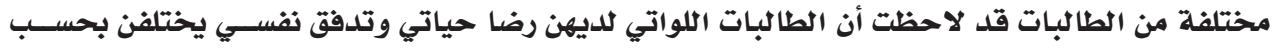

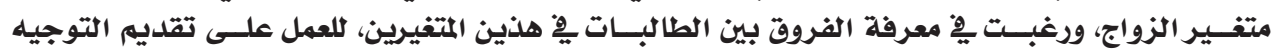

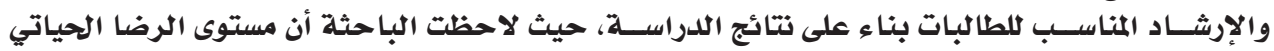

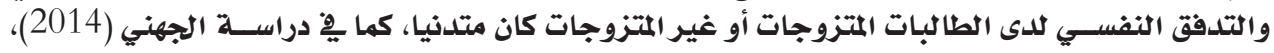

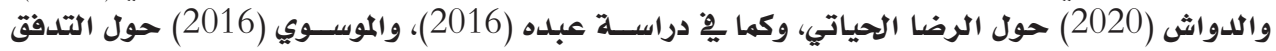

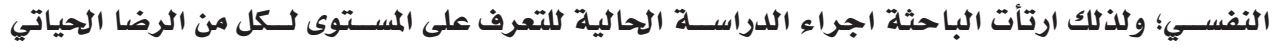

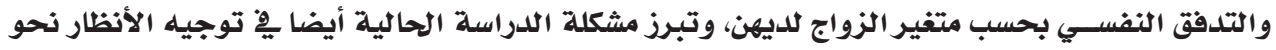

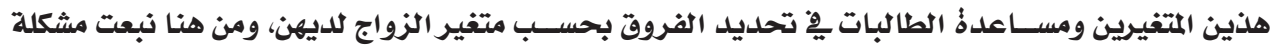
الدراسة الحالية، وهي تلدور حول السؤال الرئيسي الآتي:

ما مستوى الرضا الحياتي والتدفق النفسي للى طالبات كلية عنيزةٌ بالمملكة العربية السعودية؟ وينبثق عن هذا السؤال الأسئلة الفرعية الآتية :

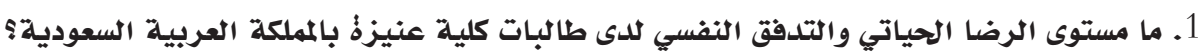

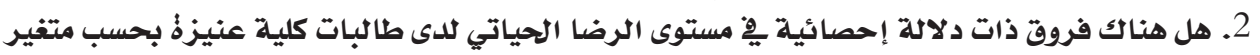

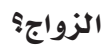
3. هل هناك فروق ذات دلاكلة إحصائية يِّ مسـتوى التدفق النفسـي لدى طالبـات كلية عنيزة بحسبـ

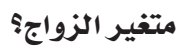

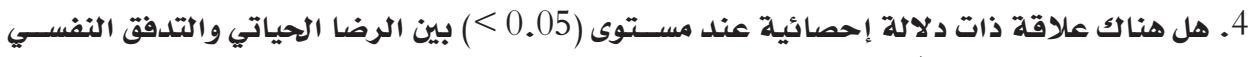

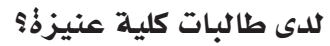
5. هل هناك فروق ذات دلالة إحصائية يِّة مســوى الرضا الحياتي لدى طالبات كلية عنيزةٌ يعزى إلى

التخصص: هنئ 6. هل هناك فروق ذات دلالة إحصائية يِّ مسـتوى التدفق النفسي لدى طالبـات كلية عنيزةٌ يعزى إلى التخصص: هل هن: فرو أهداف الدراسة: سعت الدراسة الحالية إلى تحقيق الهلدف الرئيس الآتي: التعرف على مستوى الرضا الحياتي والتدفق النفسي لدى طالبات كلية عنيزةٌ بالمملكة العربية السعودية، وينبثق عن هذا الهدف الأهداف الفرعية الآتية : 1. معرفة مستوى الرضا الحياتي والتدفق النفسي لدى طالبات كلية عنيزةٌ بالمملكة العربية السئة السعودية.

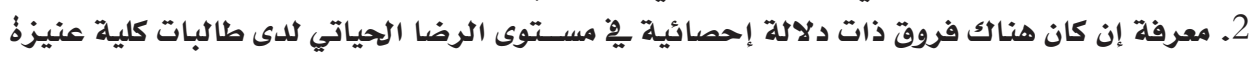
بحسب متغير الزواج. 
3. معرفة إن كان هناك فروق ذات دلالة إحصائية يِّ مسـتوى التدفق النفسي للى طالبات كلية عنيزةٌ بحسب متغير الزواج. 4. معرفة إن كان هناك علاقة ذات دلاتلة إحصائية عند مستوى (0.05 > > بين الدئ الرضا الحياتي والتدفق

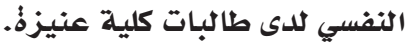
5. معرفة إن كان هناك فروق ذات دلالة إحصائية يِّ مســوى الرضا الحياتي للدى طالبات كلية عنيزةٌ يعزى إلى التخصص. 6. معرفة إن كان هناك فروق ذات دولاتلة إحصائية يِّ مسـتوى التدفق النفسي لدى طالبـات كلية عنيزةٌ يعزى إلى التخصص. أهمية الهدرالسة:

تكمن أهمية الدراسة الحالية من الناحيتين النظرية والتطبيقية على النحو الآتي:

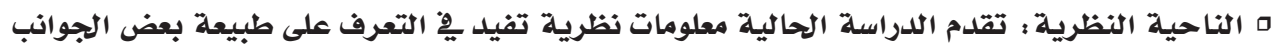

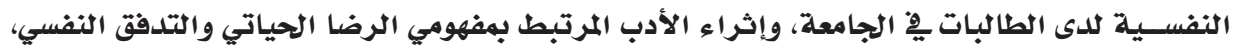

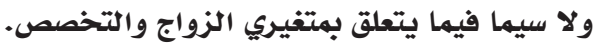

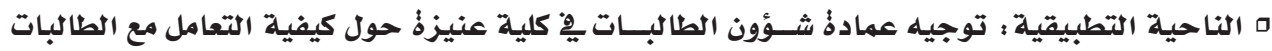

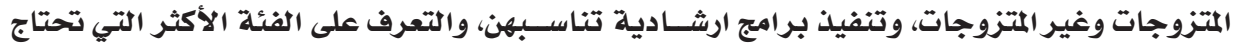

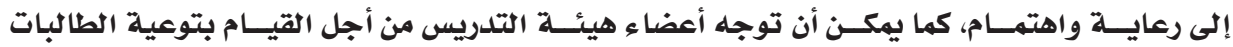

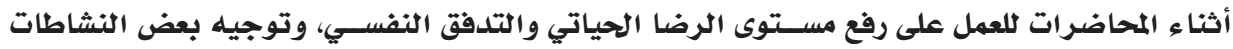

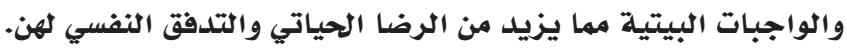

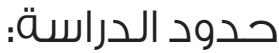
\ حلدود زمانية : تتمثل ِِّ الفصل الدراسي الثاني للعام الدراسي 2020-2021م.

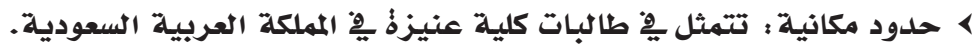

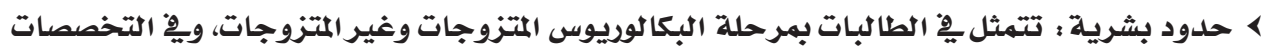
الإنسانية والهندسية التمثية.

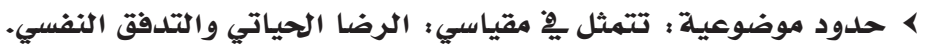

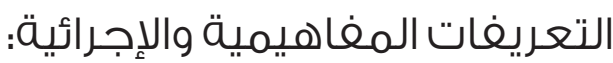

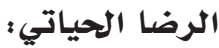

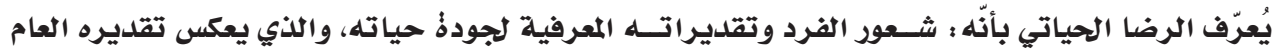
لنواح معينـة يِّ حياته، كالأسرفُ، والذات (Gilman, Ashby, Sverko, Florell, \& Varjas, 2005). التعريـف الإجرائي : هو الدرجة التي تحصل عليها طالبات كلية عنيزةٌ ِِّ مقياس الرضا الحياتي المطور يِْ هذه الدراسة.

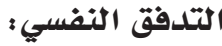

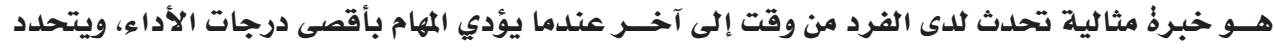

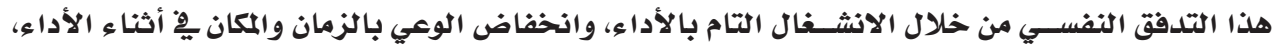

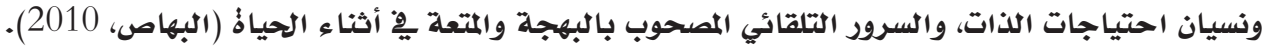
التعريـف الإجرائي : هو الدرجة التي تحصل عليها طالبات كلية عنيزةٌ ِِِّ مقياس التدفق النفسـي وهو المقياس المطور يِّ الدراسة الحالية. 
متغير الزواج: يقصد به أن الفتاذٌ تزوجت قبل تطبيق الدراسة الحالية وما زالت متزوجة إلى الآن. متفير التخصص : قســم إلى متغيرين : دراســات إنسانية وما يحتويها من تشعبـات، ودراسات هندسية وما يشملها من تشعبات. المنهفجية والتصميمج: منهجية الدراسة ؛ تم استخلام المنهج الوصفي؛ نظرا ملائمته لموضوع الدراسة.

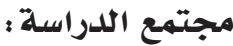

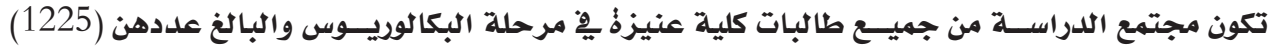

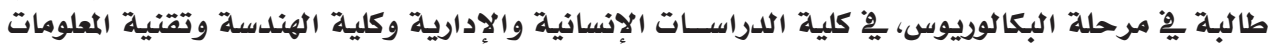
والمتزوجات وغير المتزوجات. عينة الدراسة :

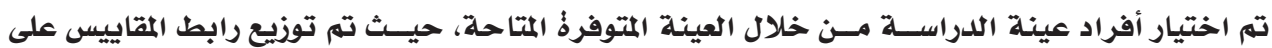

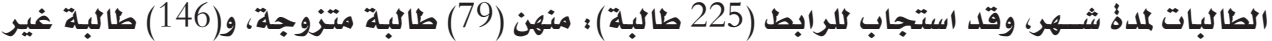

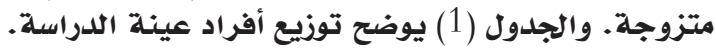
جدول (1) : توزيع افراد عينة الدراسة بحسب المتغيرات الدوات

\begin{tabular}{|c|c|c|c|}
\hline النسبـة & العدد & الفئات & المتغير \\
\hline$\% 35$ & 79 & متزوجة & متغير الزواج \\
\hline$\% 65$ & 146 & غير متزوجة & \\
\hline$\% 64$ & 143 & إنسانية & التخصص \\
\hline$\% 36$ & 82 & هندسية & \\
\hline
\end{tabular}

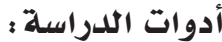

لتحقيق أهداف هذه الدراسة تم تطوير الأدوات الآتية : مقياس الرضا الحياتي، ومقياس التدفق النفسي. أولا: مقياس الرضا الحياتي:

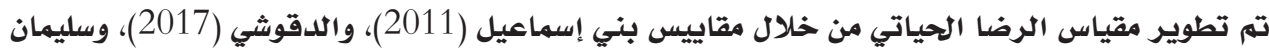

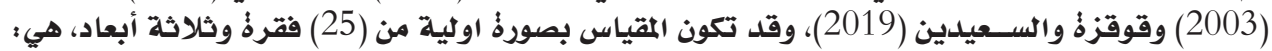

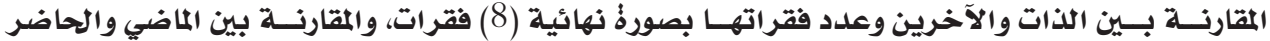

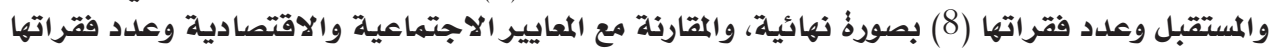

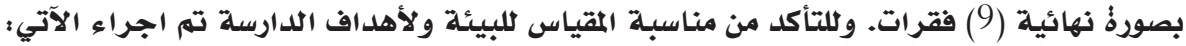
الصدق الظاهري (صدق المحكمين) :

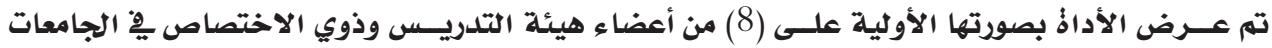

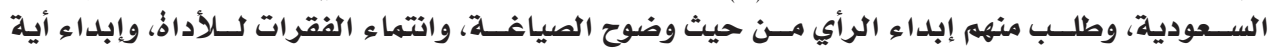

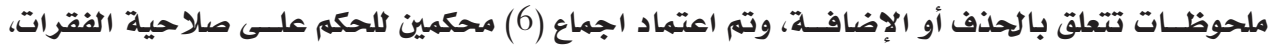

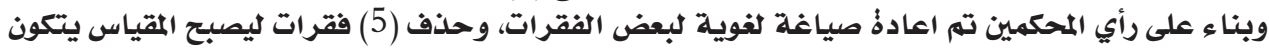

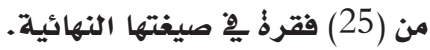
صدق البناء الداخلي (الاتساق الداخلي):

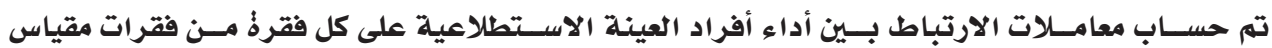

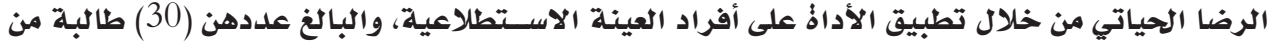




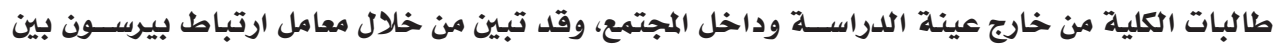

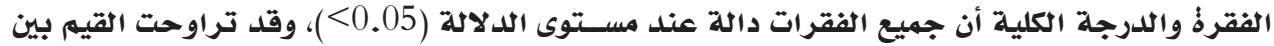

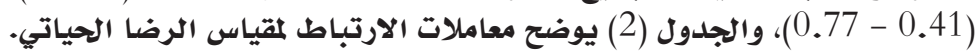

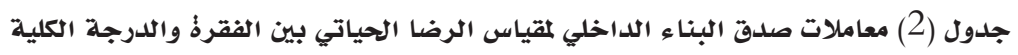

\begin{tabular}{|c|c|c|c|c|c|}
\hline معامل الارتبـاط & الرقم & معامل الارتباط & الرقم & معامل الارتبـاط & الرقم \\
\hline${ }^{* *} 0.64$ & 19 & ${ }^{* *} 0.63$ & 10 & ${ }^{*} 0.47$ & 1 \\
\hline${ }^{* *} 0.60$ & 20 & ${ }^{* *} 0.60$ & 11 & $* 0.45$ & 2 \\
\hline${ }^{* *} 0.58$ & 21 & $* * 0.68$ & 12 & ${ }^{*} 0.49$ & 3 \\
\hline${ }^{* *} 0.57$ & 22 & $* 0.47$ & 13 & ${ }^{* *} 0.58$ & 4 \\
\hline$* 0.47$ & 23 & ${ }^{* *} 0.51$ & 14 & $*_{0.41}$ & 5 \\
\hline${ }^{* *} 0.62$ & 24 & ${ }^{* *} 0.50$ & 15 & ${ }^{* *} 0.70$ & 6 \\
\hline \multirow[t]{3}{*}{${ }^{* *} 0.52$} & 25 & ${ }^{* *} 0.58$ & 16 & ${ }^{* *} 0.58$ & 7 \\
\hline & & ${ }^{* *} 0.68$ & 17 & ${ }^{* *} 0.64$ & 8 \\
\hline & & ${ }^{* *} 0.77$ & 18 & ${ }^{* *} 0.60$ & 9 \\
\hline
\end{tabular}

الثبات بطريقتي الاعادةٌ ووفق معادلة كرونباخ ألفاء

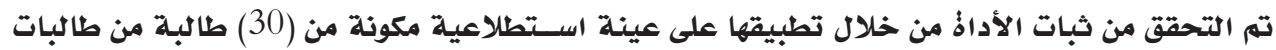

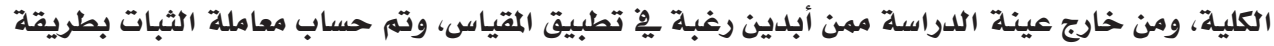

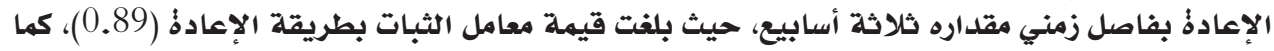

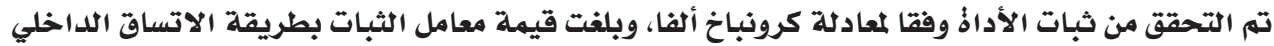

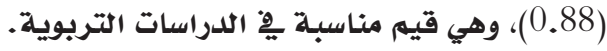
كيفية تصحيح فقرات المقياس وتفسير درجاتها :

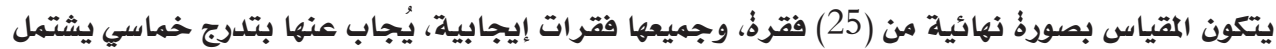

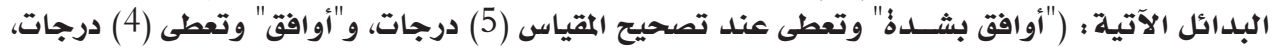

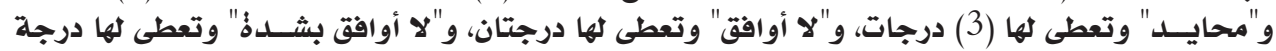

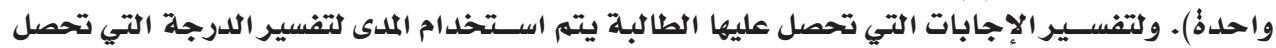

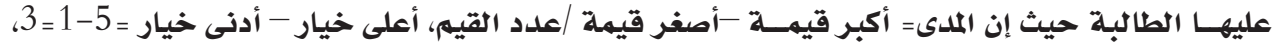

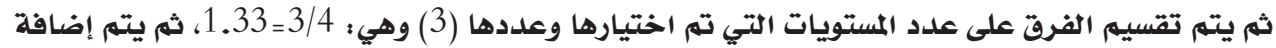

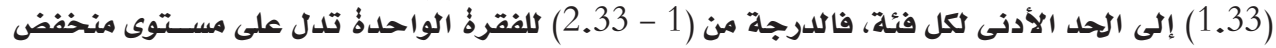

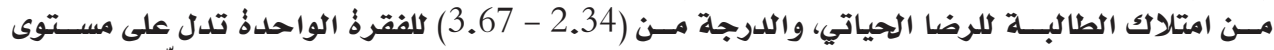

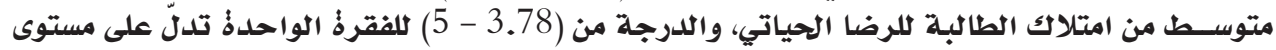

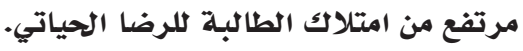

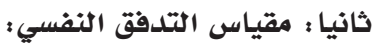

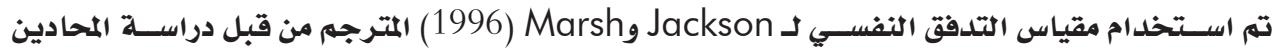

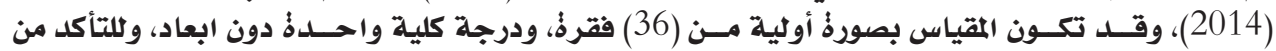

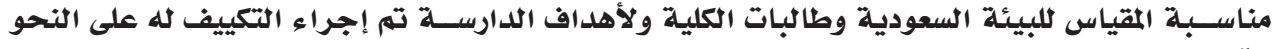
الآتي :

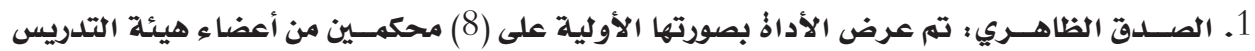

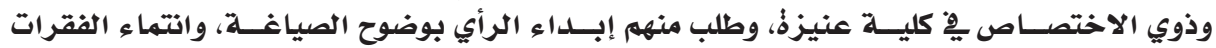




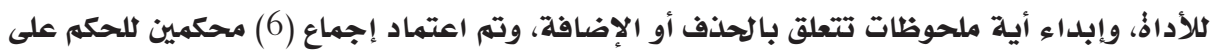

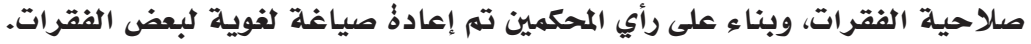

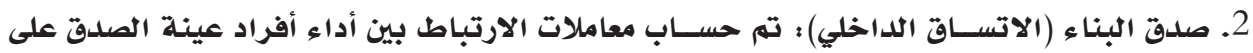

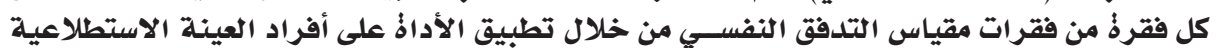

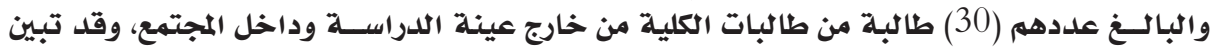

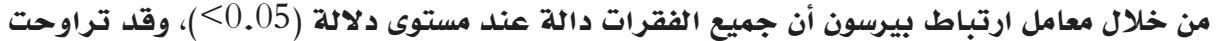

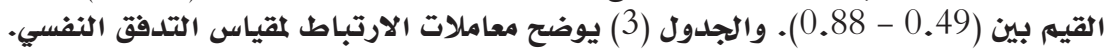

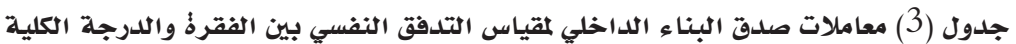

\begin{tabular}{|c|c|c|c|c|c|}
\hline معامل الارتباط & الرقم & معامل الارتباط & الرقم & معامل الارتباط & الرقم \\
\hline${ }^{* *} 0.68$ & 25 & ${ }^{* *} 0.64$ & 13 & ${ }^{* *} 0.58$ & 1 \\
\hline${ }^{* *} 0.69$ & 26 & ${ }^{* *} 0.60$ & 14 & ${ }^{* *} 0.64$ & 2 \\
\hline${ }^{* *} 0.74$ & 27 & ${ }^{* *} 0.55$ & 15 & ${ }^{* *} 0.71$ & 3 \\
\hline${ }^{* *} 0.71$ & 28 & $* * 0.58$ & 16 & ${ }^{* *} 0.75$ & 4 \\
\hline${ }^{* *} 0.73$ & 29 & ${ }^{* *} 0.60$ & 17 & $* 0.49$ & 5 \\
\hline$*^{* *} 0.78$ & 30 & ${ }^{* *} 0.68$ & 18 & ${ }^{* *} 0.66$ & 6 \\
\hline${ }^{* *} 0.68$ & 31 & ${ }^{* *} 0.88$ & 19 & ${ }^{* *} 0.70$ & 7 \\
\hline${ }^{* *} 0.60$ & 32 & ${ }^{* *} 0.82$ & 20 & ${ }^{* *} 0.77$ & 8 \\
\hline${ }^{* *} 0.68$ & 33 & ${ }^{* *} 0.78$ & 21 & ${ }^{* *} 0.78$ & 9 \\
\hline${ }^{* *} 0.64$ & 34 & ${ }^{* *} 0.83$ & 22 & ${ }^{* *} 0.70$ & 10 \\
\hline${ }^{* *} 0.58$ & 35 & ${ }^{* *} 0.68$ & 23 & ${ }^{* *} 0.80$ & 11 \\
\hline${ }^{* *} 0.52$ & 36 & ${ }^{*} 0.49$ & 24 & ${ }^{* *} 0.81$ & 12 \\
\hline
\end{tabular}

3. الثبـات بطريقتي الاعادةُ ووفق معادلة كرونباخ ألفا : تم التحقق من ثبات الأداذُ من خلال تطبيقها

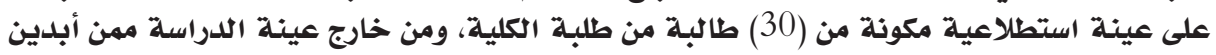

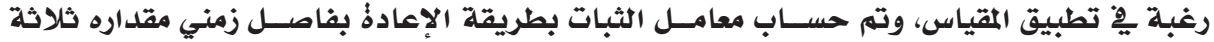

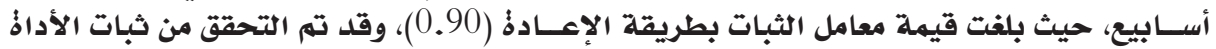

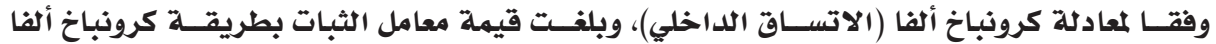

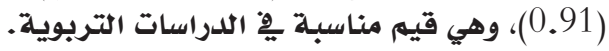
كيفية تصحيح فقرات مقياس التدفق النفسي وتفسير درجاتها :

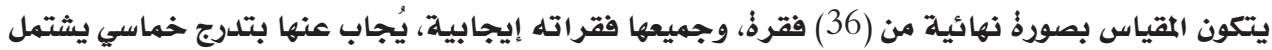

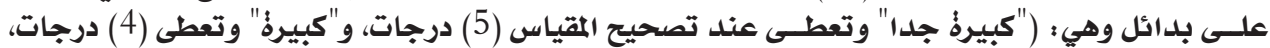

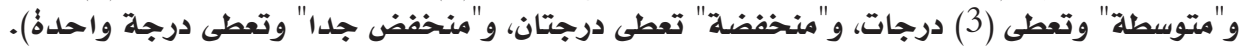

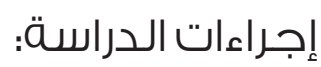

تمت الدراسة وفق الخطوات الآتية ؛

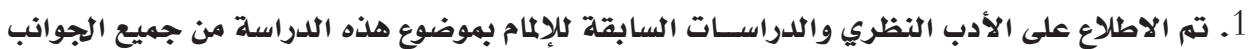

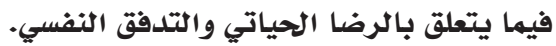

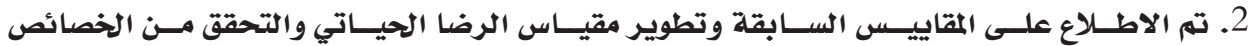

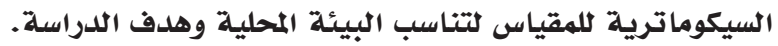


3. تم استخلم مقياس التدفق النفسي وإخضاعه للصدق والثبات ليناسب البيئة وهدف الدراسة.

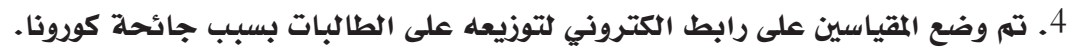

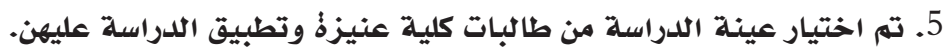

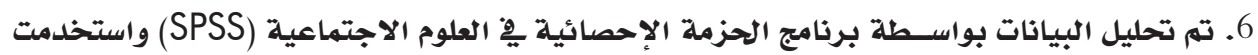
أساليب الإحصاء الوصفي والاستداتلائي للتوصل إلى إلى النتائج، ومناقشتها.

\section{نتائج الدراسة ومناقشتشها: \\ فيما يلي عرض لنتائج الدراسة ومناقشتها :}

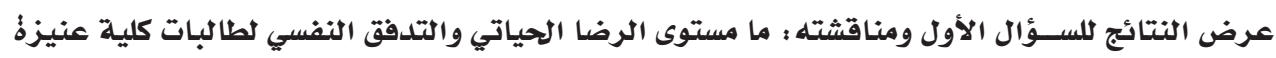

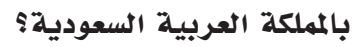

لإججابة عن هذا الســؤال تم استخدام المتوسطات الحسـابية والانحرافات المعياريـة، والجدول (4) يوضح

النتائج.

جدول (4) المتوسطات الحسابية والانحرافات المعيارية لمستوى الرضا الحياتي والتدفق النفسي

\begin{tabular}{|c|c|c|c|c|c|}
\hline الرتبـة & التقدير & الانحراف المعياري & المتوسط الحسابي & البعد & المقياس \\
\hline 3 & متوسط & 1.16 & 3.33 & المقارنة بين الذات والآخرين & الرضا الحياتي \\
\hline 1 & متوسط & 1.08 & 3.45 & المقارنة بين الماضبي والحاضر & \\
\hline 2 & متوسط & 1.23 & 3.34 & المقارنة مع المعايير الاجتماعية & \\
\hline & متوسط & 1.27 & 3.36 & الدالدجة الكلية & \\
\hline & متوسط & 1.16 & 3.53 & & النفسي \\
\hline
\end{tabular}

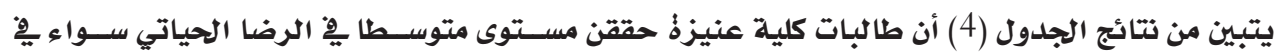

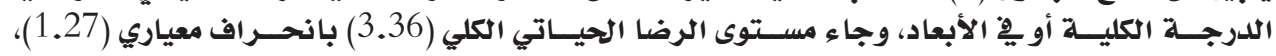

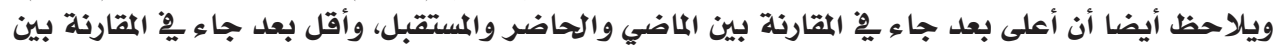

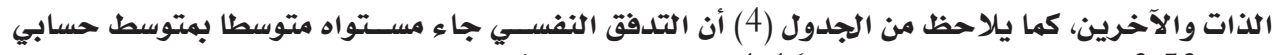

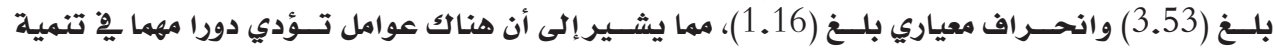

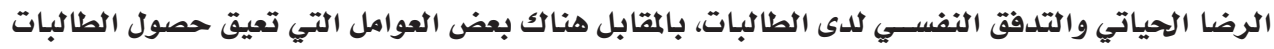

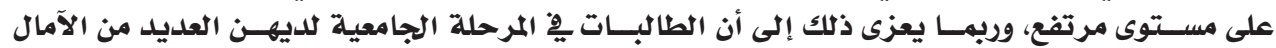

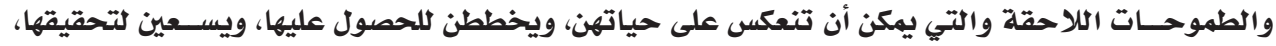

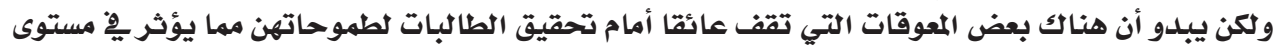

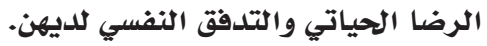

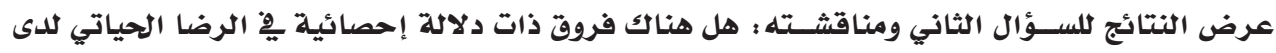

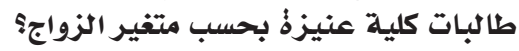

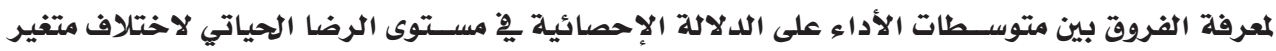

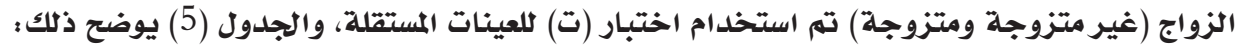


جدول (5): نتائج اختبار (ت) لمتوسطات الرضا الحياتي تببعا لمتغير الحالة الاجتماعية (غير متزوجة، متزوجة)

\begin{tabular}{|c|c|c|c|c|c|c|c|}
\hline مستوى & قيمة المتغير & الحرجية & الانحياري & المتوسط & 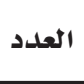 & فئة الزواج & البعد \\
\hline \multirow[t]{2}{*}{0.00} & 3.26 & 223 & 1.34 & 3.69 & 79 & متزوجة & المقارنة بين الذات \\
\hline & & & 1.00 & 3.13 & 146 & غير متزوجة & والآخرين \\
\hline \multirow[t]{2}{*}{0.01} & 2.58 & 223 & 1.30 & 3.72 & 79 & متزوجة & المقارنة بين الماضي \\
\hline & & & 0.92 & 3.29 & 146 & غير متزوجة & والحاضر والمستقبل \\
\hline \multirow[t]{2}{*}{0.01} & 2.79 & 223 & 1.46 & 3.65 & 79 & متزوجة & المقارنة مع المعايير \\
\hline & & & 1.04 & 3.13 & 146 & غير متزوجة & الاجتماعية والاقتصادية \\
\hline \multirow[t]{2}{*}{0.00} & 2.92 & 223 & 1.35 & 3.69 & 79 & متزوجة & الدرجة الكلية \\
\hline & & & 0.94 & 3.19 & 146 & غير متزوجة & \\
\hline
\end{tabular}

يظهر من الجلدول (5) وجود فروق بين الطالبات بحسـب متغير الزواج يِّ الرضا الحياتي يِّ جميع الأبعاد

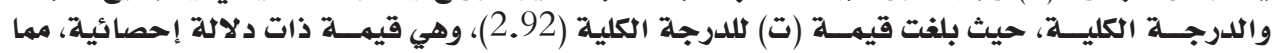

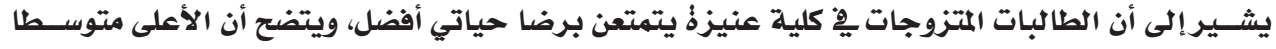

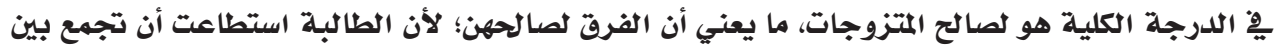

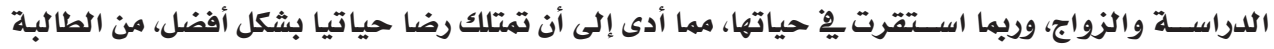

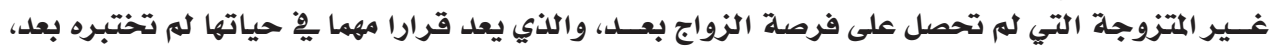

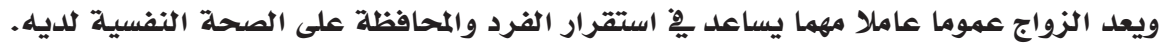

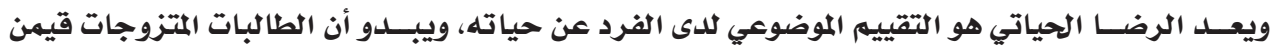

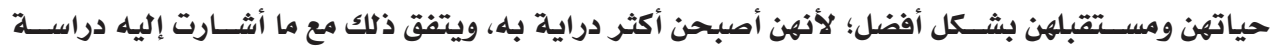
والتواحي Maltby Linley Proctor

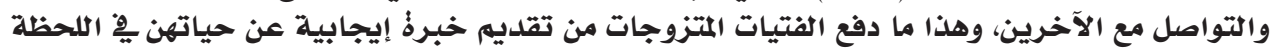

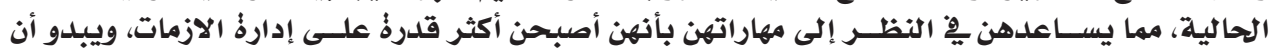

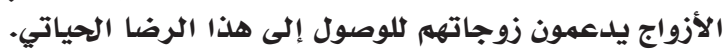

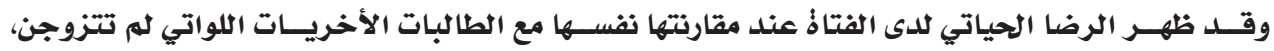

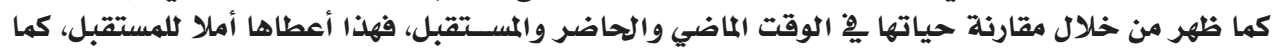

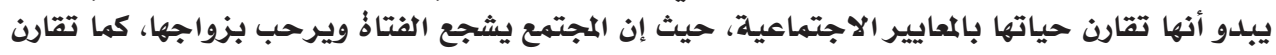

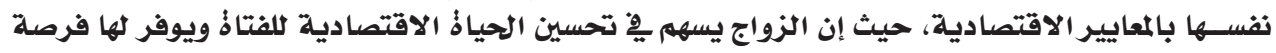
كلاكتفاء ذاتيا.

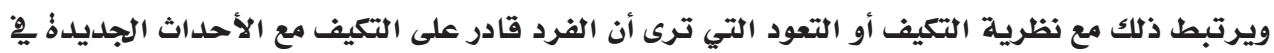

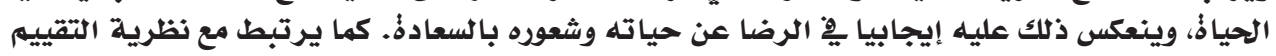

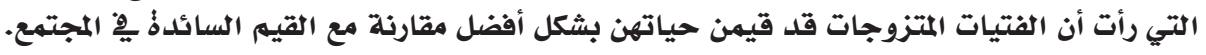
وتتفق هذه النتيجة مع نتائج دراسة . Zullig et al (2005) التي أظهرت مستوى رضا حياتيا مرتفعا. بينما تختلف مع نتائج دراسة Kwan (2010) التي أظهرت مستوى منخفضا من الرضا الحياتي.

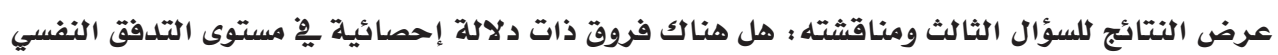

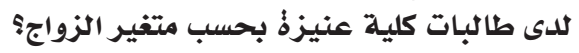

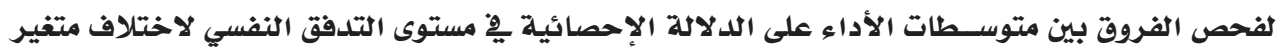

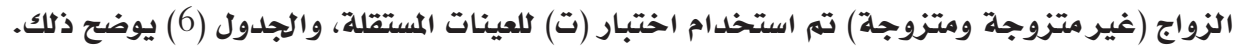


جدول (6) : نتائج اختبار (ت) لمتوسطات التدفق النفسي تبعا لمتغير الزواج (غير متزوجة ومتزوجة)

\begin{tabular}{|c|c|c|c|c|c|c|c|}
\hline الدلاكلة & قيمة المتغير & الحرجية & الانحياري & المتوسط & العدد & فئسة الزواج & المقياس \\
\hline \multirow[t]{2}{*}{0.13} & 1.58 & 223 & 1.11 & 3.62 & 79 & متزوجة & التدفق النفسي \\
\hline & & & 1.26 & 3.36 & 146 & غير متزوجة & \\
\hline
\end{tabular}

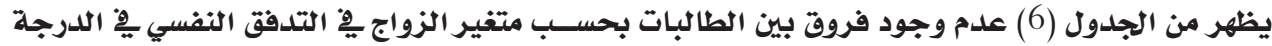

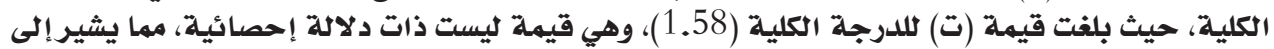

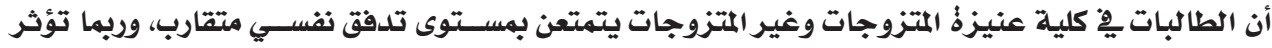

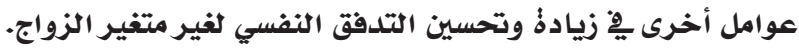

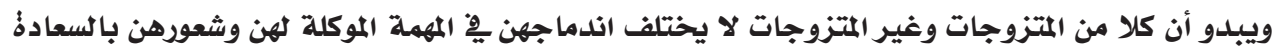

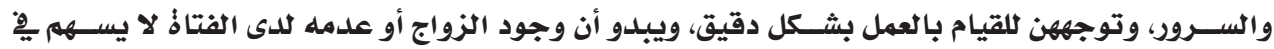

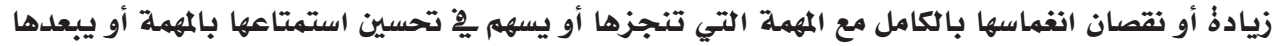

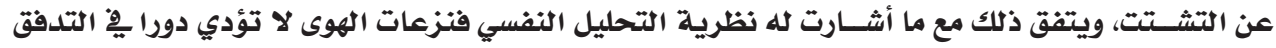

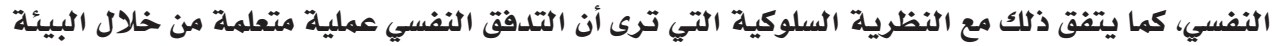

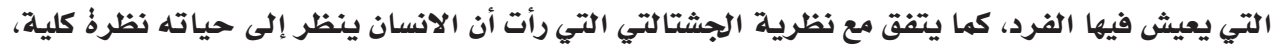

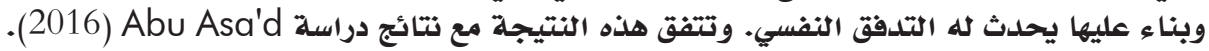

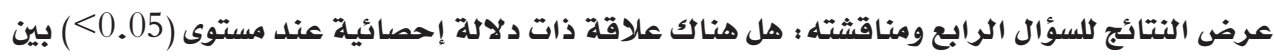

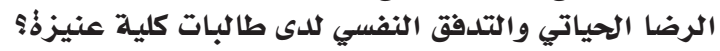

لإجابة عن الســؤال تم استخلدام معامل ارتباط بيرسون للتعرف على طبيعة العلاقة بين الرضا الرضا الحياتي

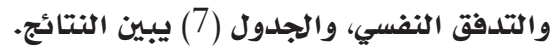

جدول (7): معامل الارتباط بين الرضا الحياتي والتدفق النفسي لدى الطالبات بِّ كلية عنيزة:

\begin{tabular}{|c|c|c|c|c|}
\hline الدرجة الكلية & الاجتماعية والاقتصادير بينة المعايير & والحقارنة بين الماضبل & 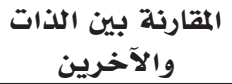 & الارتباط معامل \\
\hline${ }^{* *} 0.72$ & ${ }^{* *} 0.71$ & ${ }^{* *} 0.55$ & ${ }^{* *} 0.65$ & التدفق النفسي \\
\hline
\end{tabular}

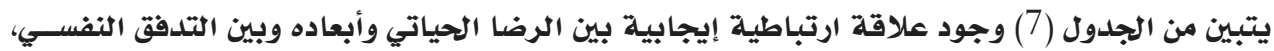

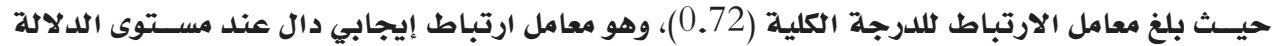

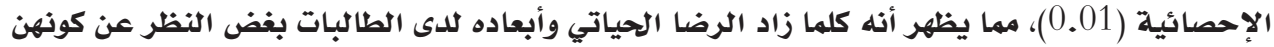

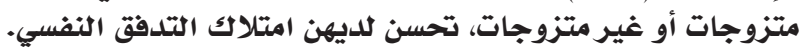

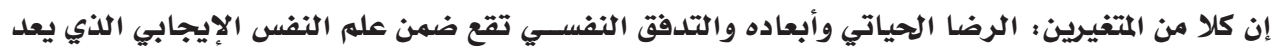

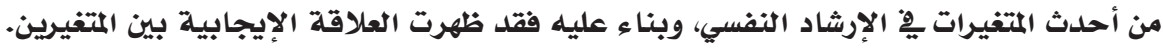

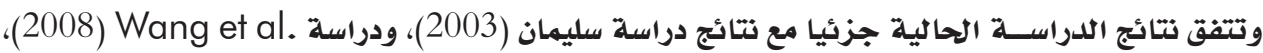

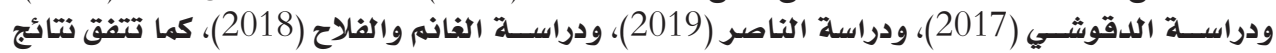
الدراسة الحالية مع نتائج دراسة الرويلي (2017)، ودراسة النياسير (2019)، ونتائج دراسة بخاريس (2019).

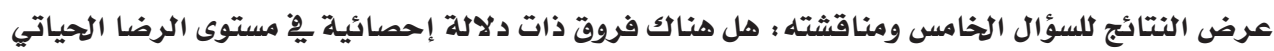

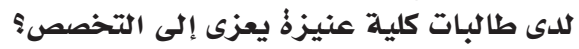

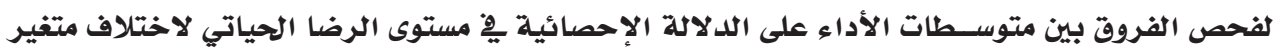

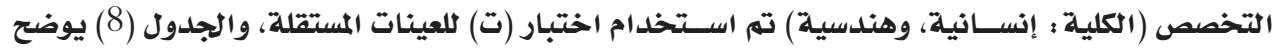




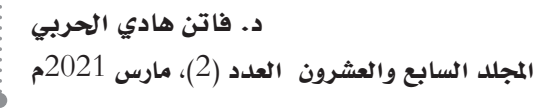

جدول (8): نتائج اختبار (ت) لمتوسطات الرضا الحياتي تِبعا لمتفير الكلية (إنسانية وهندسية)

\begin{tabular}{|c|c|c|c|c|c|c|c|}
\hline مستوى الدلائ & قيهمة المتغير & درجة الحرية & الانحياري & المتوسط & 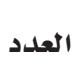 & التخصصئة & 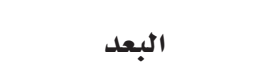 \\
\hline \multirow[t]{2}{*}{0.00} & -9.24 & 223 & 0.94 & 2.86 & 143 & إنسانية & المقارنة بين الذات \\
\hline & & & 1.03 & 4.14 & 82 & هندسية & والآخرين \\
\hline \multirow[t]{2}{*}{0.00} & -9.83 & 223 & 0.91 & 2.99 & 143 & إنسانية & المقارنة بين الماضي \\
\hline & & & 0.91 & 4.23 & 82 & هندسية & والحاضر والمستقبل \\
\hline \multirow[t]{2}{*}{0.00} & -8.93 & 223 & 1.05 & 2.84 & 143 & إنسانية & المقارنة مع المعايير \\
\hline & & & 1.07 & 4.14 & 82 & هندسية & الاجتماعية والاقتصادية \\
\hline \multirow[t]{2}{*}{0.00} & -9.75 & 223 & 0.93 & 2.89 & 143 & إنسانية & الدرجة الكلية \\
\hline & & & 0.97 & 4.17 & 82 & هندسية & \\
\hline
\end{tabular}

يظهر من الجلدول (8) وجود فروق بين الطالبات بحسب متغير التخصص يِّ الرضا الحياتي يِّ جميع الأبعاد

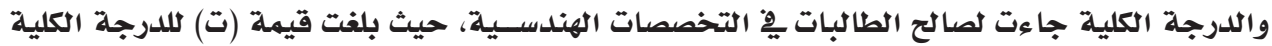

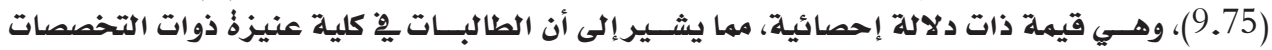

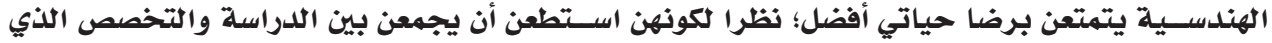

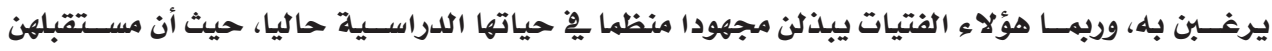

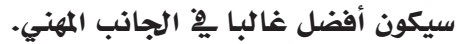

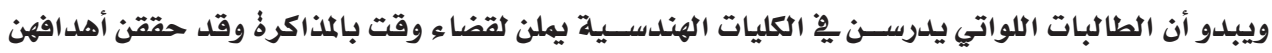

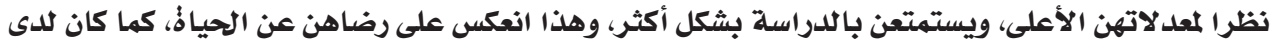

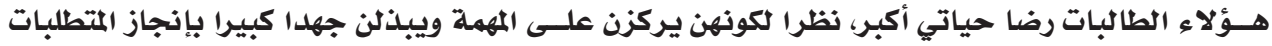

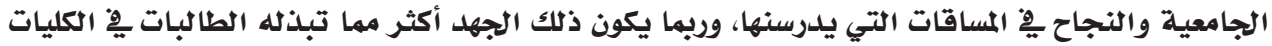

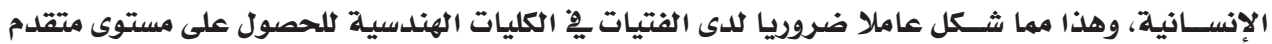

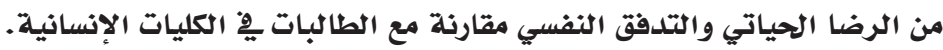

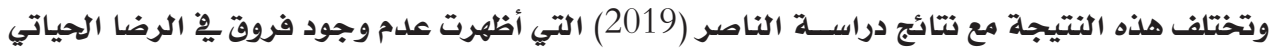

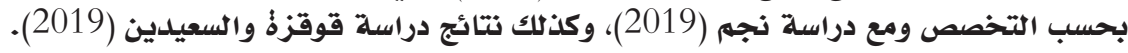
عرض النتائج للسؤال السادس ومناقشته : هل هناك فروق ذات دلاتلة إحصائية ِِِ مستوى التدفق النفسي للدى طالبات كلية عنيزةٌ تعزى إلى التى التخصصء

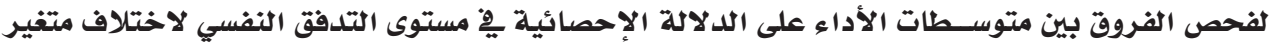

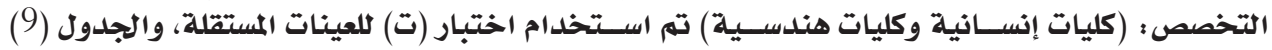
يوضح ذلك.

جدول (9): نتائج اختبار (ت) لمتوسطات التدفق النفسي تبعا لمتفير الكلية (كليات إنسانية وكليات هندسية)

\begin{tabular}{|c|c|c|c|c|c|c|c|}
\hline مستوى & قيمة المتغير & الحرجية & الانحياري افي & المتوسط & العدد & فئة الكلية & المقياس \\
\hline \multirow[t]{2}{*}{0.02} & -2.37 & 223 & 1.27 & 3.39 & 143 & إنسانية & التدفق النفسي \\
\hline & & & 0.92 & 3.77 & 82 & هندسية & \\
\hline
\end{tabular}

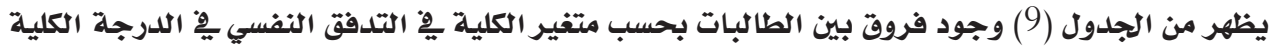

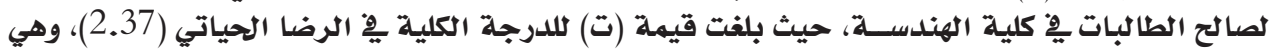

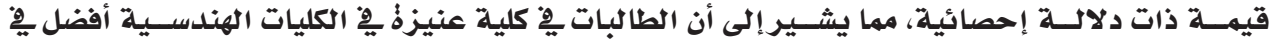


التدفق النفسي من الطالبات يْ الكليات الإنسانية؛ وربما يعود ذلك إلى اختيار التخصص التهي الذي من الممكن

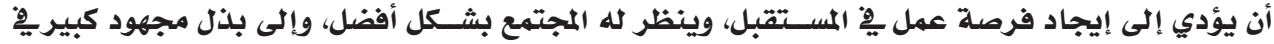

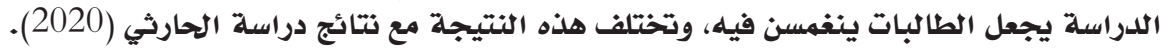

الاستنتاجات:

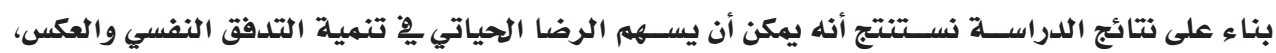

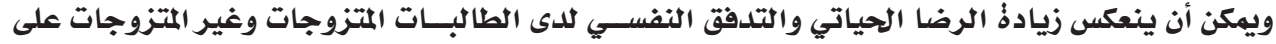

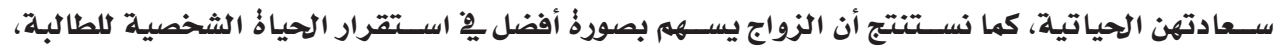

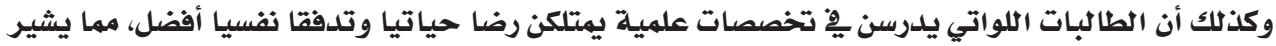

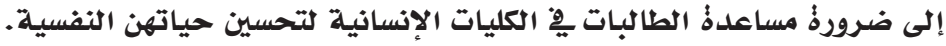

التوصيات:

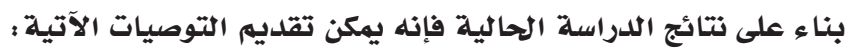

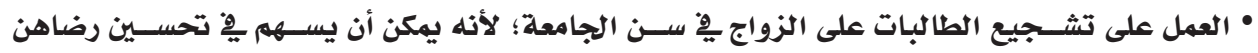
الحياتي.

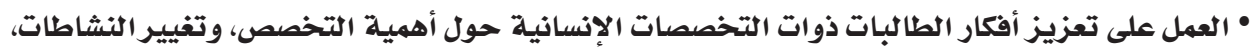

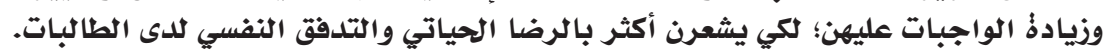

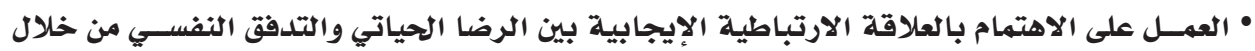
برامج ارشادية. المقترحات: • إجراء دراســة حول برنامج إرشــادي للطالبات لتحسين الرضا الحياتي والتدفق النفسي للدى عينة من

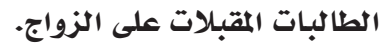

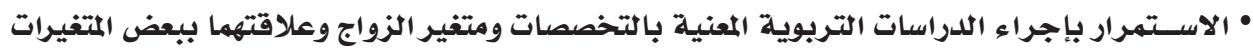

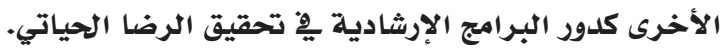

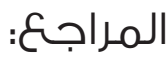

الأعسر، صفاء وكفايِ، علاء (2000)، الذكاء الوجلاني، القاهرةٌ ؛ دار قباء للطباعة والنشر.

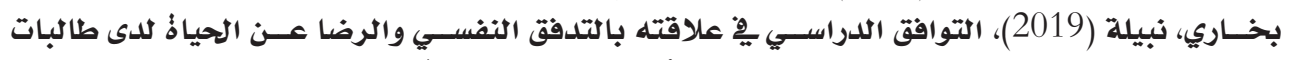

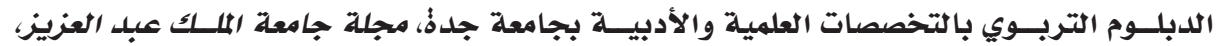
272-241، 271، (3) 27

بني إسماعيل، أحمد (2011)، الرضا عن الحياءة للى المراهقين وعلاقتته بأساليب التنشئة الأسربية والرضا

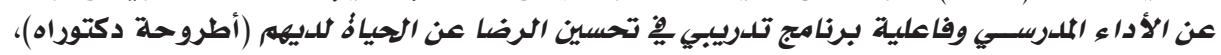
الجامعة الأردنية، عمان.

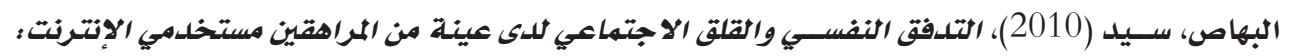

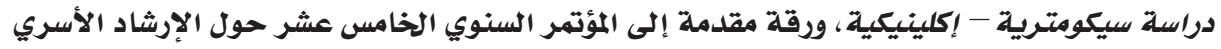

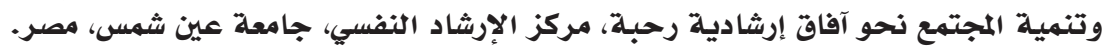

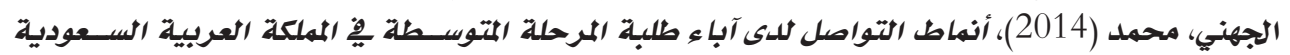
وعلاقتها بالرضا الحياتي لهه (رسالة ماجستير)، جامعة مؤتة، التهاء الأردن.

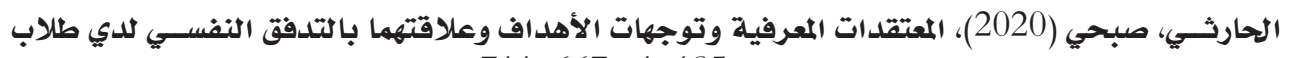

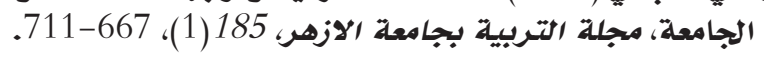




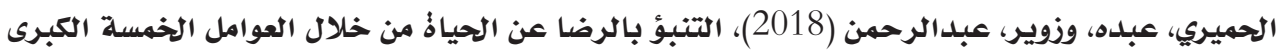

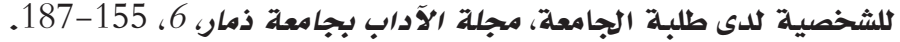

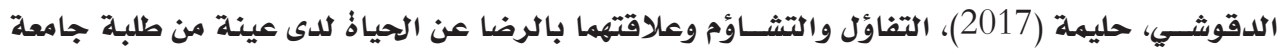

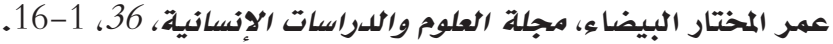

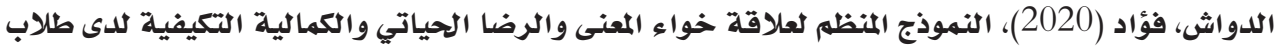

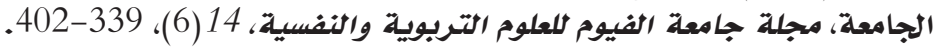

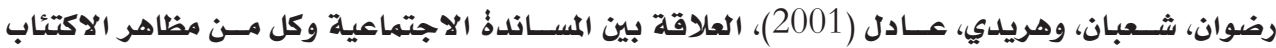

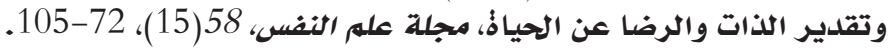

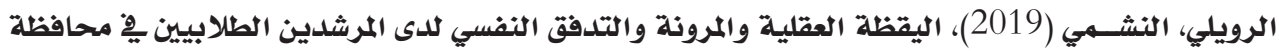

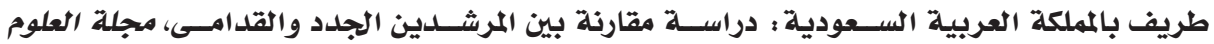
التربيوية والنفسية، 3(7)، بالمبرية، 114-130.

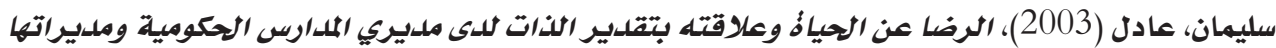

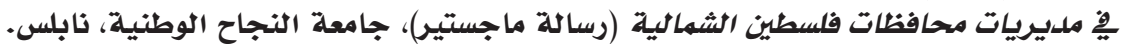

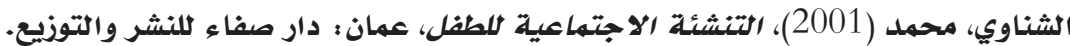

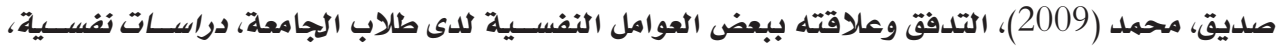
357-313، 19 19 19

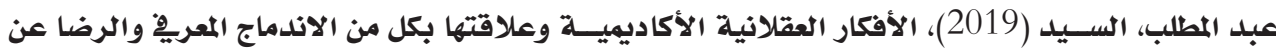

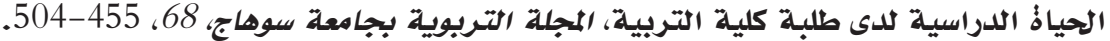

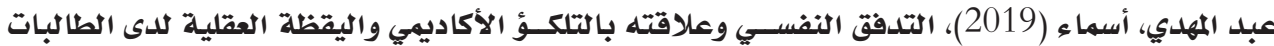

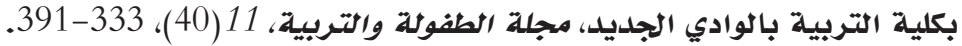

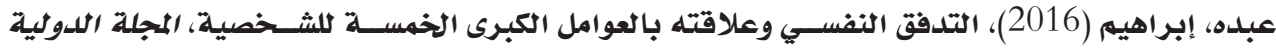

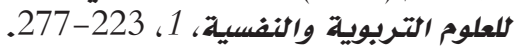

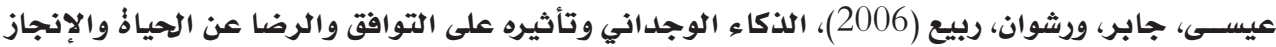

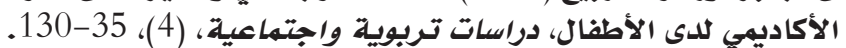

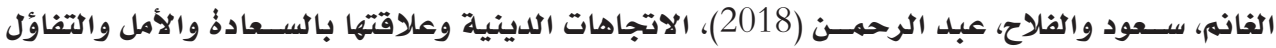

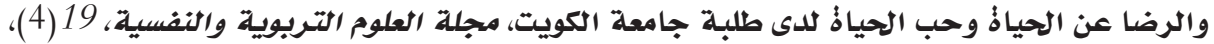
.139-109

الفنجري، حسـن (2000)، التغيرات الاجتماعية يْ محافظة الوادي الجديد وعلاقتتها بالتدفق النفسي يِ

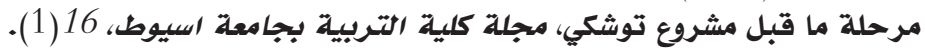

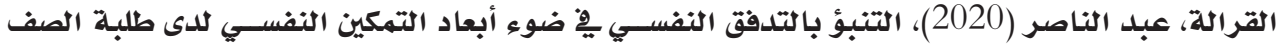

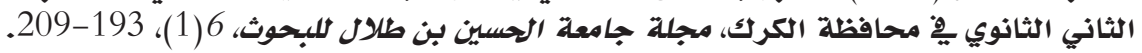

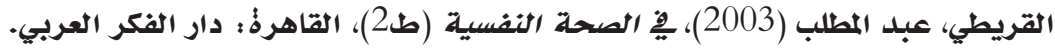

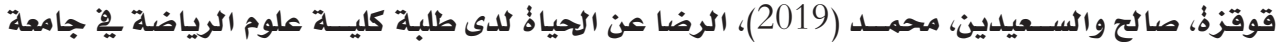

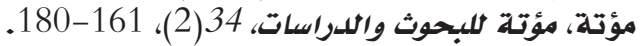

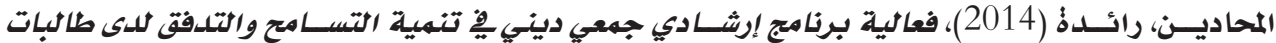

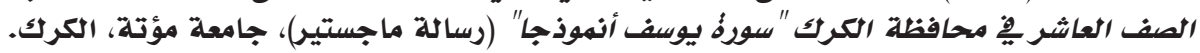

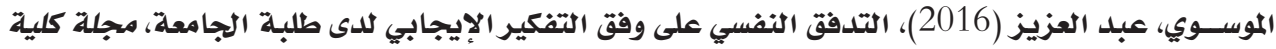

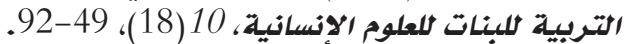

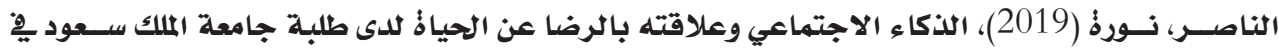

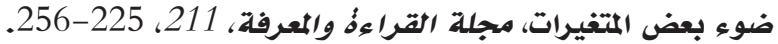




$$
\text { دا داتلن هادي الحربي السابع والعشرون العدد (2)، مارس 2021م }
$$

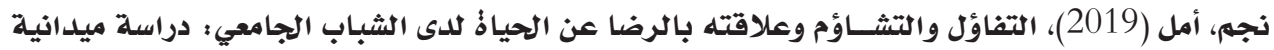

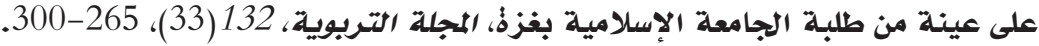

Abu Asa'd, A. A. E. (2016). Level of psychological flow and its relationship with psychological flexibility among Mu'tah University students in Al-Karak Governorate/South Jordan. Research on Humanities and Social Sciences, 6(6), 100-108.

Ayyash-Abdo, H., \& Alamuddin, R. (2007). Predictors of subjective well-being among college youth in Lebanon. The Journal of Social Psychology, 147(3), 265-284.

Chiang, L. M. (2010). The development of a leisure and life satisfaction scale for outpatient (LLSSOP) leisure activity programs in lowa (Doctoral dissertation). University of Northern lowa, Cedar Falls, lowa.

Chin-Sheng, W., \& Chiou, W. B. (2007). The motivations of adolescents who are addicted to online games: A cognitive perspective. Adolescence, 42(165), 179.

Diener, E. (2000). Subjective well-being: The science of happiness and a proposal for a national index. American Psychologist, 55(1), 34.

Diener, E., \& Ryan, K. (2009). Subjective well-being: A general overview. South African Journal of Psychology, 39(4), 391-406.

Easterlin, R. A. (2001). Life cycle welfare: Evidence and conjecture. The Journal of Socio-Economics, 30(1), 31-61.

Gilman, R., Ashby, J. S., Sverko, D., Florell, D., \& Varjas, K. (2005). The relationship between perfectionism and multidimensional life satisfaction among Croatian and American youth. Personality and Individual Differences, 39(1), 155-166.

Jackson, S. A., \& Marsh, H. W. (1996). Development and validation of a scale to measure optimal experience: The flow state scale. Journal of Sport and Exercise Psychology, 18(1), 17-35.

King, J. J. (2005). Gender ideology: Impact on dual-career couples' role strain, marital satisfaction, and life satisfaction (Doctoral dissertation). Texas A\&M University, College Station, Texas.

Kwan, Y. K. (2010). Life satisfaction and self-assessed health among adolescents in Hong Kong. Journal of Happiness Studies, $77(3), 383-393$.

Normal, D. (1996). Optimal flow. Arts Education Policy Review, 97(4), 35-38.

Proctor, C. Linley, P \& Maltby, J. (2008). Youth life satisfaction: Review of the literature. Journal of Happiness Studies, 10(8)902-922.

Suldo, S. M., \& Huebner, E. S. (2006). Is extremely high life satisfaction during adolescence advantageous? Social Indicators Research, 78(2), 179-203. 


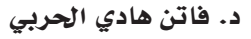

المجلد السابع والعشرون العدد (2)، مارس 2021 فادي الحربي

Treistman, D. (2004). Work-family conflict and life satisfaction in female graduate students: Testing mediating and moderating hypotheses (Doctoral dissertation). University of Maryland, Maryland.

Veenhoven, R. (2001). What we know about happiness. Paper presented at the dialogue on Gross National Happiness, 14-15 January, Woudschoten, Zeist, The Netherlands.

Wang, E. S. T., Chen, L. S. L., Lin, J. Y. C., \& Wang, M. C. H. (2008). The relationship between leisure satisfaction and life satisfaction of adolescents concerning online games. Adolescence, 43(169), 177-84

Zullig, K. J., Valois, R. F., Huebner, E. S., \& Drane, J. W. (2005). Associations among family structure, demographics, and adolescent perceived life satisfaction. Journal of Child and Family Studies, 14(2), 195-206.

\section{Arabic References in Roman Scripts:}

Abdel Muttalib, El-Sayed (2019). Al'afkar aleaqlaniat al'akadimiat waealaqatuha bikulin min alaindimai almaerifii walrida ean alhayaat aldirasiat ladaa talbat kuliyat altarbiati, Almajalat Altarbawiat Bijamieat Suhai, 68, 455504.

Abdo, Ibrahim (2016). Altadafuq alnafsiu waealaqatuh bialeawamil alkubraa alkhamsat lilshakhsiati, Almajalat Aldawliat Lileulum Altarbawiat Walnafsiati, 1, 223-277.

Abdul-Mahdi, Asma (2019). Altadafuq alnafsiu waealaqatuh bialtalakuw al'akadimii walyaqazat aleaqliat ladaa altaalibat bikuliyat altarbiat bialwadi aljadidi, Majalat Altufulat Waltarbiati, 1 1(40), 333-391.

Al-Assar, Safaa wa Kafafi, Alaa (2000). Aldhaka' alwijdani, Alqahirata: Dar Qaba' Liltibaeat Walnashri.

Al-Bahas, Syed (2010). Altadafuq alnafsiu walqalaq alaijtimaeiu ladaa eayinat min almurahiqin mustakhdimi al'iintirnta: Dirasat sikumitriat - 'iiklinikiatan, Waraqat muqadimat 'iilaa almutamar alsanawii alkhamis eashar hawl Al'iirshad Al'usarii Watanmiat Almujtamae Nahw Afaq 'lirshadiat Rahbata, Markiz Al'iirshad Alnafsi, Jamieat Eayn Shams, Misr.

Al-Daqoushi, Halima (2017). Altafawul waltashawum waealaqatuhuma bialrida ean alhayaat ladaa eayinat min talabat Jamieat Omar Almukhtar Albayda', Majalat Aleulum Waldirasat Al'iinsaniati, 36, 1-16.

Al-Dawash, Fouad (2020). Alnamudhaj almunazam liealaqat khiwa' almaenaa walrida alhayatii walkamaliat altakayufiat ladaa tulaab aljamieati, Majalat Jamieat Alfayuwm Lileulum Altarbawiat Walnafsiati, 14(6), 339-402. 


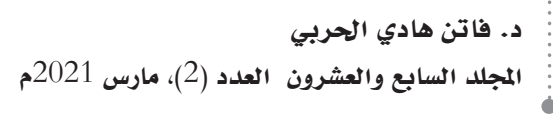

Al-Fangari, Hasan (2000). Altaghayurat alaijtimaeiat fi muhafazat alwadi aljadid waealaqatiha bialtadafuq alnafsii fi marhalat ma qabl mashrue tushki, Majalat Kuliyat Altarbiat Bijamieat Asyut, 16(1).

Al-Ghanim, Saud wa Al-Falah, Abdul Rahman (2018). Aliatijahat aldiyniat waealaqatuha bialsaeadat wal'amal waltafawul walrida ean alhayat wahubu alhayaat ladaa talabat Jamieat Alkuayta, Majalat Aleulum Altarbawiat Walnafsiati, 19(4), 109-139.

Al-Harthy, Sobhi (2020). Almuetaqadat almaerifiat watawajuhat al'ahdaf waealaqatuhuma bialtadafuq alnafsii ladaya tulaab aljamieati, Majalat Altarbiat Bijamieat Alaizhr, 185(1), 667-711.

Al-Humairi, Abdo, wa Zuwer, Abdul Rahman (2018). Altanabuw bialrida ean alhayaat min khilal aleawamil alkhamsat alkubraa lilshakhsiat ladaa talabat aljamieati, Majalat Aladab Bijamieat Dhimar, 6, 155-187.

Al-Juhani, Muhammad (2014). 'Anmat altawasul ladaa aba' talabat almarhalat almutawasitat fi Almamlakat Alearabiat Alsaeudiat waealaqatiha bialrida alhayaatii lahum (Risalat majistir), Jamieat Mutata, Al'urdunn.

Al-Mahadin, Raeda (2014). Faeaaliat barnamaj 'ïrshadiin jameiun diniun fi tanmiat altasamuh waltadafuq ladaa talbaant alsafi aleashir fi Muhafazat Alkark "Surat Yusif anmwdhiaan" (Risalat majistir), Jamieat Mutata, Alkarka.

Al-Moussawi, Abdulaziz (2016). Altadafuq alnafsiu ealaa wifq altafkir al'iijabii ladaa talabat aljamieati, Majalat Kuliyat Altarbiat Lilbanat Lileulum Al'iinsaniati, 10(18), 49-92.

Al-Nasser, Noura (2019). Aldhaka' aliajtimaeiu waealaqatuh bialrida ean alhayaat ladaa talabat jamieat almalik sueud fi daw' baed almutaghayirati, Majalat Alqira'at Walmaerifati, 21 1, 225-256.

Al-Qarala, Abdel-Nasser (2020). Altanabuw bialtadafuq alnafsii fi daw' 'abead altamkin alnafsii ladaa talabat alsafi althaani althaanawii fi Muhafazat Alkarka, Majalat Jamieat Alhusayn bin Talal Lilbuhuth, 6(1), 193-209.

Al-Quraiti, Abdel-Muttalib (2003). Fi alsihat alnafsia (Taba'a 2), Alqahirata: Dar Alfikr Alearabii.

Al-Ruwaili, Al-Nashmi (2019). Alyaqazat aleaqliat walmurunat waltadafuq alnafsiu ladaa almurshidin altulaabiiyn fi muhafazat tarif bialmamlakat alearabiat alsueudiati: Dirasat muqaranat bayn almurshidin aljudud walqudamaa, Majalat Aleulum Altarbawiat Walnafsiati, 3(7), 114-130.

Al-Shennawi, Muhammad (2001). Altanshiat aliajtimaeiat liltifli, Amman: Dar Safa' Lilnashr Waltawziei.

Bani Ismail, Ahmed (2011). Alrida ean alhayaat ladaa almurahiqin waealaqatih bi'asalib altanshiat al'usariat walrida ean al'ada' almadrasii wafaeiliat barnamai tadribiin fi tahsin alrida ean alhayaat ladayhim ('Utaruhat dukturah), Aljamieat Al'urduniyatu, Amman. 
Bukhari, Nabila (2019). Altawafuq aldirasiu fi ealaqatih bialtadafuq alnafsii walrida ean alhayaat ladaa talibat aldiblum altarbawii bialtakhasusat aleilmiat wal'adabiat bijamieat jidat, Majalat Jamieat Almalik Eabd Aleaziza, 27(3), 241-272.

Issa, Jaber, Wa Rashwan, Rabie (2006). Aldhaka' alwijdaniu watathiruh ealaa altawafuq walrida ean alhayat wal'iinjaz al'akadimii ladaa al'atfali, Dirasat Tarbawiat Wajtimaeiatun, (4), 35-130.

Najm, Amal (2019). Altafawul waltashawum waealaqatuh bialrida ean alhayaat ladaa alshabab aljamieii: Dirasat maydaniat ealaa eayinat min talabat Aljamieat Al'iislamiat bi Ghaza, Almajalat Altarbawiati, 132(33), 265-300.

Quqazah, Saleh wa Al-Saeedin, Muhammad (2019). Alrida ean alhayaat ladaa talbat kuliyat eulum alriyadat fi Jamieat Mutata, Mutatan Lilbuhuth Waldirasati, 34(2), 161-180.

Radwan, Shaban wa Haridi, Adel (2001). Alealaqat bayn almusanadat aliaijtimaeiat wakulin min mazahir alaiktiaab wataqdir aldhaat walrida ean alhayati, Majalat Eilm Alnafs, 58(15), 72-105.

Siddiq, Muhammad (2009). Altadafuq waealaqatuh bibaed aleawamil alnafsiat ladaa tulaab aljamieati, Dirasat Nafsiat, 19(2), 313-357.

Suleiman, Adel (2003). Alrida ean alhayaat waealaqatih bitaqdir aldhaat ladaa mudiri almadaris alhukumiat wamudiratiha fi mudiriaat muhafazat filastin alshamalia (Risalat majistir), Jamieat Alnajah Alwataniati, Nabuls. 